\title{
Dynamic Hedging of Synthetic CDO Tranches: Bridging the gap between theory and practice
}

\author{
Areski Cousin $^{1}$ and Jean-Paul Laurent ${ }^{2}$ \\ March, 23, 2010
}

\begin{abstract}
This paper intends to provide insights about the topical issue of risk managing synthetic CDOs. We stand in the grey zone between mathematical finance and financial econometrics, between academic and market practitioners approaches. We chose to first present two scholar models, each of them leading to perfect replication of CDO tranches with credit default swaps. Though they rely upon rather simplistic assumptions and are built upon different premises, they lead to similar hedge ratios. We also stress that the study of the hedging issue in these two approaches involves the same basic theoretical ingredients. We then discuss various problems related to the use of such models in designing hedging strategies for CDO tranches and back testing or assessing hedging performance. At this stage, it appears that model based hedging strategies do help in the risk management process. Even though correlation markets had to face serious tests, more data related to short term maturity equity tranche spreads and plain CDS are required to discriminate against competing modelling approaches.
\end{abstract}

Keywords: CDOs, hedging, replication strategies, market completeness, Markovian contagion models, hedging efficiency, back-testing.

JEL: G13

\section{Introduction}

The theory is when you know everything and nothing works. The practice is when everything works and nobody knows why. We have put together the theory and practice: there is nothing that works... and nobody knows why! Albert Einstein

The risk management and the hedging of CDOs and related products are topics of tremendous importance, yet much has to be done both on theoretical and empirical grounds. The risks at hand are usually split into different categories, which may sometimes overlap, such as credit spread and default risks, correlation and contagion risks. These will be the centre of our discussion. This does not mean that these risks are orthogonal, for instance an increase in credit spreads is likely to increase the occurrence of defaults; due to contagion effects, the arrival of defaults may trigger jumps in credit spreads and changes in the dependence structure between default times, etc. For simplicity, we will focus on the hedging of synthetic CDO tranches on reference indexes such as iTraxx Europe or CDX.NA.IG. The 2007-2008 crisis also drove attention to liquidity, counterparty risks and related issues such as recovery risk, collateral management, downgrading of guarantors, basis risks which we will briefly address. Focus was concentrated on the risks within senior tranches and tail risks. Conversely, the 2005 crisis, was driven by some rather specific event, namely the widening of spreads in the automobile sector and led to some disruptions between equity and junior mezzanine tranches, associated with idiosyncratic gamma risks and unwinding of positive carry trades.

\footnotetext{
${ }^{1}$ ISFA Actuarial School, Université Lyon 1, Université de Lyon, 50 avenue Tony Garnier, 69007, Lyon, France, areski.cousin@univ-lyon1.fr, http://www.acousin.net

${ }^{2}$ ISFA Actuarial School, Université Lyon 1, Université de Lyon, 50 avenue Tony Garnier, 69007, Lyon, France, and BNP-Paribas, laurent.jeanpaul@free.fr, http://laurent.jeanpaul.free.fr
} 
Before going any further with hedging of CDO tranches, we need to account for the way models are used and the reliance upon market data. We will take as an example the modelling of dependence of default dates of names within a credit portfolio. A quick glance at the literature should convince us that it is a key point in disentangling the hedging puzzle.

Ingenuously, we could think of a probabilistic construction of default times that matches the actual behaviour of defaults, spreads, tranche quotes, assume that this probabilistic construction could be perfectly determined and that given some set of well identified hedging instruments, we end-up with, maybe, a self-financing replicating strategy for CDO tranches. Then, the hedging and risk management puzzle would be solved thanks to good computational skills and eventually some kind of algorithmic hedging would consecrate the reign of robots and expel from the trading rooms, all sorts of quantitative analysts, traders and risk managers, which are nowadays considered by a number of bright minds as an hybridization of dummies and crooks abusing and misusing mathematics. This would likely be the final stage in the evolutionary process and the "end of history" as far as credit derivatives are concerned.

Actually, we subsequently describe two scholar models, one belonging to the category of contagion models, the other one to that of structural models, so that within the model, perfect hedging strategies of CDO tranches can be computed. This is somehow good news since credit might eventually no longer be the ugly duckling of mathematical finance..

However, when it comes to putting models at work, the issue appears trickier that it looked at first sight and the picture becomes blurred. Let us assume for a while that the above ingenuous modeller with superlative skills reaches the Holy Grail ${ }^{3}$ of designing the true probabilistic description of default times. Such a probabilistic model is likely to involve a small set of correlation parameters (say it is a parametric model). There should be some parameters such that CDO tranches of any maturity, attachment and detachment point should be matched at one fell swoop at any time. But, even if we were to restrict to CDO quotes for a given maturity, all parametric models we have in mind fail the perfect calibration test and are thus misspecified ${ }^{4}$. As a consequence, one cannot predict from scratch what would be the hedging performance of such a model, assuming that perfect replication of CDO tranches is meaningful within the above model, i.e., some form of market completeness is achieved. For instance, the structural model associated with an underlying multivariate Brownian motion would most likely fail to price junior, mezzanine and senior tranches with some flat correlation matrix ${ }^{5}$. In terms of model specification, this means that the model is rejected with certainty, since it predicts deterministic relationships across prices, which are inconsistent with observed data.

In order to circumvent this issue, the usual way is to relax some assumptions, such as constant correlation, Brownian increments in asset prices, introducing some clustering effects through

\footnotetext{
${ }^{3}$ For instance, some academics tend to think that the use of a Gumbel, Clayton or a t-copula would have avoided the pitfalls of the Gaussian copula approach. We refer to Burtschell et al. (2009), Cousin and Laurent (2008a), Cousin and Laurent (2008c) or Gregory and Laurent (2008) for reviews of a number of popular pricing approaches.

${ }^{4}$ For this reason, some authors have considered models, where the expected tranche loss surface is an input parameter. While, consistency with traded tranche quotes at a given point in time is fulfilled by construction, this approach has its own difficulties. It is not clear whether the loss dynamics will be consistent with the recalibration process. Moreover, as is known in equity markets, a dynamic model (say a local volatility model) that is consistent with market data can be associated with poor hedging properties.

${ }^{5}$ By "flat" correlation matrix, we mean that pairwise correlation parameters are constant across names.
} 
stochastic volatility ${ }^{6}$. This is not priceless. First, it leads to increased complexity both on numerical grounds and in understanding the relevant dynamics. Then, one might lose some suitable properties of the simpler model, such as the existence of self-financing replicating strategies, which leads to difficulties in designing optimal hedging strategies (in incomplete markets). Parsimony is for sure an important property of a relevant model. When it comes to models where the number of parameters is large, sometimes even larger than the number of liquid calibrating prices, one can rightfully question the usefulness of such an approach. Also, one may feel growing unease looking at this data driven inference, where the purpose is to find some probability distribution over default times which matches observed data ${ }^{7}$. Looking at the world of defaults through such glasses and entering a risk management process from that premise is a perilous adventure.

The culmination of the inverse problem of model design from market prices leads to some nonparametric approaches such as the local volatility model in the equity field and its local intensity counterpart in the credit domain (see Cont and Minca (2008), Cont, Deguest. and Kan (2009)). This relies upon some hidden though not innocuous assumptions such as the absence of simultaneous defaults. Moreover, given some sparse market data, one usually needs to parameterize the local intensity, for instance assuming piecewise linear dependence upon the number of defaults. The devil is in this kind of detail since credit deltas may actually greatly depend upon such kind of arbitrary numerical scheme. For instance, when computing a default hedge in a local intensity loss model, one would need to assess the change of the loss intensity after a first default, something which cannot be directly inferred from CDO tranche quotes. It is worth remarking that these implied dynamics are usually associated with a one dimensional Markov process, which is quite convenient for simplicity, but is likely to shrink the risks to be handled.

If one model as described above would be deemed worthy, it should be time consistent. For instance, in the case of a parametric model, calibrated parameters should remain constant over time. As this is never the case, one actually deals with some unknown dynamics, possibly some kind of incompleteness due to jumps in calibrated parameters. It is also likely that implied parameters are actually correlated with the underlying "assets", say credit default swaps spreads, thus one has to choose either to compute the total or partial derivative to derive hedge ratios. We just wanted to stress that given that recalibration process, the possibly desirable properties of the initial theoretical model are likely to be lost.

Moreover, given any model, market practice consists in bumping initial conditions rather than computing derivatives with respect to the underlying assets (assuming a Markovian framework). For instance, when using an intensity model, instead of computing sensitivities with respect to short-term intensities ${ }^{8}$, one will shift the credit curves, recalibrate the model and look for the change in the prices of CDO tranches. The computed deltas have little connection if any, with the theoretical deltas.

\footnotetext{
${ }^{6}$ Here, we have implicitly assumed that the new model embeds the older one. One could either switch to a distinct approach. In the credit field, there are actually different seeds, say for simplicity such as structural and reduced-form models. Some footbridges have already been thrown over the gap and we will mention the useful effort to integrate the probabilistic framework. Much as the claim that stock prices are semimartingales, we will be left with a very small set of testable restrictions as the counterpart of our broad mathematical view, which is clearly some issue for the purpose of building useful models. As French say, "qui trop embrasse, mal étreint".

${ }^{7}$ The best use of such an approach is to provide a consistent interpolation procedure to compute consistent prices of less liquid prices for accounting purpose.

${ }^{8}$ Assuming that name predefault intensities follow a multivariate diffusion process.
} 
Self financing replicating strategies are usually set up within a theoretical context and, when applied in a market context, have no reason to lead to a replication of the promised payoff. This is not surprising then, that the corresponding theoretical models are not dealt with by market practitioners according to the theory, when it comes to computing hedging ratios. Hedging strategies derived from a hypothetical complete markets framework can be seen as a convenient benchmark for further analysis, but there is no guarantee that these would perform better than a model with poorer theoretical or dynamical properties. This is not to say that financial models are useless; actually empirical evidence tends to show that model based hedging outperforms statistical based hedging and leads to a significant reduction in risk. Let us also point out that hedging performance can be dramatically improved whenever a CDO book is statically hedged, i.e., CDO tranches are hedged with other similar CDO tranches, and the dynamic hedging with credit default swaps addresses only residual risks.

Trying to encompass such a large topic, we chose to discuss, in a first part, the theory that underpins the two most documented and understood pricing and hedging models as far as CDO tranches are concerned. In a second part, we focus on a number of methodological and implementation issues related to the use of such models ${ }^{9}$.

\section{I) Hedging of CDO tranches: theoretical issues and perspectives}

As far as the laws of mathematics refer to reality, they are not certain. As far as they are certain, they do not refer to reality. Albert Einstein

\section{I.1 Default times and aggregate loss process}

Throughout this chapter, we consider a credit portfolio with $n$ risky obligors. The corresponding default times $\tau_{1}, \ldots, \tau_{n}$ are assumed to be non negative and finite random variables defined on a common probability space $(\Omega, A, P)$, where $P$ is the historical probability measure. The evolution of default states in the portfolio is driven by default indicator processes $N_{t}^{1}=1_{\left\{\tau_{1} \leq t\right\}}, \ldots, N_{t}^{n}=1_{\left\{\tau_{n} \leq t\right\}}$. That is, for any name $i=1, \ldots, n, N_{t}^{i}=1$ if firm $i$ has defaulted before $t$ or $N_{t}^{i}=0$ otherwise.

Let us remark that default times can be reinterpreted in terms of a marked point process, i.e., a sequence of ordered credit events $\left(T_{i}\right)_{i \in I}$ satisfying $T_{i}<T_{i+1}$ associated with some other random elements $\left(Z_{i}\right)_{i \in I}$, called marks, containing further information about defaults, such as the identity of names which have defaulted. In the general case where simultaneous defaults are possible, each mark may contain a set of names and the size of the mark space is possibly equal to $2^{n}$. This has a direct consequence on the completeness of the market, since in that case, the hedging of defaultable claim would require non standard instruments whose default payments are contingent to the arrival of joint defaults. Conversely, when simultaneous defaults are precluded, the size of the mark space is reduced to $n$. In what follows, we consider that the latter assumption is satisfied.

\footnotetext{
${ }^{9}$ We refer to Laurent et al. (2007), Cousin and Laurent (2008b), Cousin et al. (2009) for contributions related to this chapter.
} 
Regarding the pricing of CDO tranches, the key quantity is the fractional cumulative loss process $L_{t}=\frac{1}{n} \sum_{i=1}^{n}\left(1-R_{i}\right) N_{t}^{i}$, where $R_{1}, \ldots, R_{n}$ denote the recovery rates in case of default of names $i=1, \ldots, n$. The loss process $L_{t}$ is thus an increasing right-continuous pure jump process.

\section{I.2 CDO tranche cash-flows}

A synthetic CDO tranche is a structured product based on an underlying portfolio of equally weighted reference entities subject to credit risk ${ }^{10}$. The cash-flows associated with a synthetic CDO tranche only depend upon the realized path of the cumulative losses on the reference portfolio. Default losses on the credit portfolio are split along some thresholds (attachment and detachment points) and allocated to the various tranches. A CDO tranche with attachment point $a$, detachment point $b$ and maturity $T$ is essentially a bilateral contract between a protection seller and a protection buyer. We describe below the cash-flows associated with the default payment leg (payments received by the protection buyer) and the premium payment leg (payments received by the protection seller).

\section{Default payments leg}

The protection seller agrees to pay the protection buyer default losses each time they impact the tranche $[a, b]$ of the reference portfolio. More precisely, the cumulative default payment $L_{t}^{[a, b]}$ on the tranche $[a, b]$ is equal to zero if $L_{t} \leq a$, to $L_{t}-a$ if $a \leq L_{t} \leq b$ and to $b-a$ if $L_{t} \geq b$. Let us remark that $L_{t}^{[a, b]}$ has a call spread payoff with respect to $L_{t}$ and can be expressed as $L_{t}^{[a, b]}=\left(L_{t}-a\right)^{+}-\left(L_{t}-b\right)^{+}$. Default payments are simply the increment of $L_{t}^{[a, b]}$, i.e., there is a payment of $L_{t}^{[a, b]}-L_{t-}^{[a, b]}$ from the protection seller at every jump time of $L_{t}^{[a, b]}$ occurring before contract maturity $T$. If $r_{t}$ denotes the continuously compounded default free interest rate and $B_{t}=\exp \left(-\int_{0}^{t} r_{s} d s\right)$ the associated discount factor, the discounted payoff corresponding to default payments can written as:

$$
\int_{0}^{T} B_{t} d L_{t}^{[a, b]}=\sum_{i=1}^{n} B_{\tau_{i}}\left(L_{\tau_{i}}^{[a, b]}-L_{\tau_{i}-}^{[a, b]}\right) 1_{\left\{\tau_{i} \leq T\right\}} .
$$

\section{Premium payments leg}

The protection buyer has to pay the protection seller a periodic premium (quarterly for standardized indexes) based on a fixed spread or premium $S$ and proportional to the current outstanding nominal of the tranche $b-a-L_{t}^{[a, b]}$. Let us denote by $t_{i}, i=1, \ldots, I$ the premium payment dates with $t_{I}=T$ and by $\Delta_{i}$ the length of the $i^{\text {th }}$ period $\left[t_{i-1}, t_{i}\right]$ (in fractions of a year and with $\left.t_{0}=0\right)$. The CDO premium payments are equal to $S \Delta_{i}\left(b-a-L_{t_{i}}^{[a, b]}\right)$ at regular

\footnotetext{
${ }^{10}$ We refer the reader to Meissner (2008), De Servigny and Jobst (2007) textbooks or Kakodkar et al. (2006) for a detailed analysis of the CDO market and credit derivatives cash-flows.
} 
payment dates $t_{i}, i=1, \ldots, I$. Moreover, when a default occurs between two premium payment dates and when it affects the tranche, an additional payment (the accrued coupon) must be made at default time to compensate the change in value of the tranche outstanding nominal. For example, if name $j$ defaults between $t_{i-1}$ and $t_{i}$, the associated accrued coupon is equal to $S\left(\tau_{j}-t_{i-1}\right)\left(L_{\tau_{j}}^{[a, b]}-L_{\tau_{j^{-}}}^{[a, b]}\right)$. Eventually, the discounted payoff corresponding to premium payments can be expressed as:

$$
\sum_{i=1}^{I}\left(B_{t_{i}} S \Delta_{i}\left(b-a-L_{t_{i}}^{[a, b]}\right)+\int_{t_{i-1}}^{t_{i}} B_{t} S\left(t-t_{i-1}\right) d L_{t}^{[a, b]}\right) .
$$

\section{I.3 Filtration}

A CDO tranche position is typically hedged by entering opposite positions on a portfolio of liquid hedging instruments such as credit default swaps referencing names in the reference portfolio. Other examples of hedging instruments are credit default swaps standardized indices or standard tranches referencing these indices. The composition of the hedging portfolio needs to be regularly adjusted as the market environment quickly evolves. In order to tackle the hedging issue in a dynamical way, one needs to specify how information is progressively disclosed to the market.

We first investigate the framework of contagion intensity models for which the hedging of CDO tranches can be fully described in a dynamical way. In a second step, we will try to establish some connections with other hedging approaches.

As we consider the hedging of CDO tranches whose cash-flows are driven by the realization of defaults in the portfolio, the smallest filtration one shall consider may include information associated with the arrival of defaults. Let us denote by $H_{t}^{i}=\sigma\left(N_{s}^{i}, s \leq t\right), i=1, \ldots, n$, $H_{t}=\underset{i=1}{\vee} H_{i, t}$. The filtration $\left(H_{t}\right)_{t \in \mathbb{R}^{+}}$is referred to as the natural filtration associated with the default times.

We moreover assume that there exist some $\left(P, H_{t}\right)$-intensities associated with the counting processes $N_{t}^{i}, i=1, \ldots, n$, i.e., there exist some (non negative) $H_{t}$ - predictable processes $\alpha^{1, P}, \ldots, \alpha^{n, P}$, such that the processes defined by

$$
M_{t}^{i, P}:=N_{t}^{i}-\int_{0}^{t} \alpha_{s}^{i, P} d s, i=1, \ldots, n
$$

are $\left(P, H_{t}\right)$ - martingales. This implies in particular that, for any name $i=1, \ldots, n$, the default intensity process $\alpha^{i, P}$ must vanish after the default of name $i$, i.e., $\alpha_{t}^{i, P}=0$ on the set $\left\{t>\tau_{i}\right\}$. Let us recall that simultaneous defaults are precluded in this framework, i.e., $P\left(\tau_{i}=\tau_{j}\right)=0$ for $i \neq j$. 


\section{I.4 Predictable representation theorem and equivalent changes of probability measure}

The main theoretical tool for the analysis of the hedging issue is a predictable representation theorem (see Brémaud (1981) textbook, chapter III). It states that, for any $H_{T}$-measurable $P$-integrable random variable $A$, there exists some $H_{t}$-predictable processes $\theta^{1}, \ldots, \theta^{n}$ such that:

$$
A=E^{P}[A]+\sum_{i=1}^{n} \int_{0}^{T} \theta_{s}^{i}\left(d N_{s}^{i}-\alpha_{s}^{i, P} d s\right)
$$

This result provides a way to express any default-contingent payoff as a sum of stochastic integrals with respect to the fundamental martingales $M_{t}^{i, P}, i=1, \ldots, n$.

Interestingly, it can also be used to characterize equivalent changes of probability measure. From the predictable representation theorem, one can show that any Radon-Nikodym derivative $\zeta$ (that is, a strictly positive martingale with expectation equal to 1 ) can be written as

$$
d \zeta_{t}=\zeta_{t-} \sum_{i=1}^{n} \pi_{t}^{i} d M_{t}^{i, P}, \zeta_{0}=1
$$

where $\pi^{1}, \ldots, \pi^{n}$ are some $H_{t}$-predictable processes. Conversely, the unique solution of the latter stochastic differential equation is the local martingale (Doléans-Dade exponential)

$$
\zeta_{t}=\exp \left(-\sum_{i=1}^{n} \int_{0}^{t} \pi_{s}^{i} \alpha_{s}^{i, P} d s\right) \prod_{i=1}^{n}\left(1+\pi_{\tau_{i}}^{i}\right)^{N_{t}^{i}} .
$$

Note that, in order that $\zeta$ is indeed a non-negative local martingale, one needs that $\pi_{t}^{i}>-1$. Moreover, the process $\zeta$ is a true martingale under some integrability conditions on the $\pi^{i}$, s (e.g., $\pi^{1}, \ldots, \pi^{n}$ bounded) or if $E^{P}\left[\zeta_{t}\right]=1$ for any $t$. We can now define a new probability measure $Q$ from $P$ thanks to the Radon-Nikodym derivative $\zeta$ :

$$
\left.d Q\right|_{H_{t}}=\left.\zeta_{t} d P\right|_{H_{t}} .
$$

Eventually, it can be proved that under this new probability measure $Q$ the default intensity $\alpha^{i, Q}$ of $\tau_{i}$ is proportional to the default intensity $\alpha^{i, P}$ of $\tau_{i}$ under $P$. More precisely, for any name $i=1, \ldots, n$ the process $M^{i, Q}$ defined by

$$
M_{t}^{i, Q}:=N_{t}^{i}-\int_{0}^{t}\left(1+\pi_{s}^{i}\right) \alpha_{s}^{i, P} d s
$$

is a $\left(Q, H_{t}\right)$ - martingale. We refer the reader to the book chapter by Cousin et al. (2009) for more details on the construction of equivalent changes of probability measure in this framework.

\section{I.4 Hedging instruments}

For the sake of simplicity, let us assume for a while that instantaneous digital credit default swaps are traded on the names. An instantaneous digital credit default swap on name $i$ traded at $t$, provides a payoff equal to $d N_{t}^{i}-\alpha_{t}^{i} d t$ at $t+d t$. The quantity $d N_{t}^{i}$ corresponds to the payment on the default leg and $\alpha_{t}^{i} d t$ is the (short term) premium on the default swap. Note that considering such instantaneous digital default swaps rather than actually traded credit 
default swaps is not a limitation of our purpose. This can rather be seen as a convenient choice of basis from a theoretical point of view ${ }^{11}$.

We assume that contractual spreads $\alpha^{1}, \ldots, \alpha^{n}$ are non negative processes adapted to the filtration $H_{t}$ of default times. The natural filtration of default times can thus be seen as the relevant information on economic grounds. Since we deal with the filtration generated by default times, the credit default swap premiums are deterministic between two default events. Therefore, we restrain ourselves to a market where only default risk occurs and credit spreads themselves are driven by the occurrence of defaults. In our simple setting, there is no specific credit spread risk. This corresponds to the framework of Bielecki et al. (2007) and Bielecki, Crépey et al. (2007).

For simplicity, we further assume that (continuously compounded) default-free interest rates are constant and equal to $r$. Given some initial investment $V_{0}$ and some $H_{t}$-predictable processes $\delta^{1}, \ldots, \delta^{n}$ associated with some self-financed trading strategy in instantaneous digital credit default swaps, we attain at time $T$ the payoff $V_{0} e^{r T}+\sum_{i=1}^{n} \int_{0}^{T} \delta_{s}^{i} e^{r(T-s)}\left(d N_{s}^{i}-\alpha_{s}^{i} d s\right)$. By definition, $\delta_{s}^{i}$ is the nominal amount of instantaneous digital credit default swap on name $i$ held at time $s$. This induces a net cash-flow of $\delta_{s}^{i} \times\left(d N_{s}^{i}-\alpha_{s}^{i} d s\right)$ at time $s+d s$, which has to be invested in the default-free savings account up to time $T$.

\section{I.5 Pricing measure and perfect hedging}

We define a pricing measure as a probability measure $Q$ equivalent to $P$ and such that, under $Q$, the default intensities associated with default times are exactly equal to the short-term credit spreads $\alpha^{1}, \ldots, \alpha^{n}$. From the previous analysis on equivalent changes of probability measure, one can readily describe the (unique) pricing measure.

Firstly, it is natural to assume that $\left\{\alpha_{t}^{i}>0\right\} \stackrel{P-a . s .}{=}\left\{\alpha_{t}^{i, P}>0\right\}$ for all time $t$ and all name $i=1, \ldots, n$. Indeed, from the absence of arbitrage opportunity, the short-term premium on a given name $i$ at any date $t$ is positive $\left(\alpha_{t}^{i}>0\right)$ if and only if the default of that name is likely to occur at time $t\left(\alpha_{t}^{i, P}>0\right)$. As a consequence, for any $i=1, \ldots, n$, the process $\pi^{i}$ defined by

$$
\pi_{t}^{i}=\left(\frac{\alpha_{t}^{i}}{\alpha_{t}^{i, P}}-1\right)\left(1-N_{t-}^{i}\right)
$$

is a $H$-predictable process such that $\pi^{i}>-1$.

Secondly, following the previous analysis, the process $\zeta$ defined by

$$
\zeta_{t}=\exp \left(-\sum_{i=1}^{n} \int_{0}^{t} \pi_{s}^{i} \alpha_{s}^{i, P} d s\right) \prod_{i=1}^{n}\left(1+\pi_{\tau_{i}}^{i}\right)^{N_{t}^{i}}
$$

\footnotetext{
${ }^{11}$ Of course, it is possible to compute credit deltas with respect to traded credit default swaps in this framework. We refer the reader to Laurent et al. (2007) for more details on this point.
} 
is a true Radon-Nikodym derivative. Moreover, under the probability $Q$ build from $P$ thanks to the equivalent change of probability measure $\left.d Q\right|_{H_{t}}=\left.\zeta_{t} d P\right|_{H_{t}}$, the processes

$$
M_{t}^{i}:=N_{t}^{i}-\int_{0}^{t} \alpha_{s}^{i} d s, i=1, \ldots, n
$$

are $\left(Q, H_{t}\right)$-martingales. In particular the short term credit spreads $\alpha^{1}, \ldots, \alpha^{n}$ are the intensities of default times under the new probability $Q$. Given a specification of default intensities under the historical probability measure $P$, it is important to remark that the pricing measure is uniquely determined by the dynamics of short-term credit spreads. In the rest of the study, we will work under the pricing measure $Q$.

Unsurprisingly, the possibility of perfect hedging is related to a martingale representation theorem under the pricing measure. Let us consider some $H_{T}$-measurable $Q$-integrable payoff $A$. Since $A$ depends upon the default indicators of the names up to time $T$, this encompasses the cases of CDO tranches and basket default swaps, provided that recovery rates are deterministic ${ }^{12}$. It is possible to show that the predictable representation theorem described above also holds under the probability $Q$. There exists some $H_{t}$-predictable processes $\theta^{1}, \ldots, \theta^{n}$ such that:

$$
A=E^{Q}[A]+\sum_{i=1}^{n} \int_{0}^{T} \theta_{s}^{i}\left(d N_{s}^{i}-\alpha_{s}^{i} d s\right) .
$$

Let us remark that, due to the predictable property of the $\theta$ 's, the processes defined by $t \rightarrow \int_{0}^{t} \theta_{s}^{i}\left(d N_{s}^{i}-\alpha_{s}^{i} d s\right)$ are also $\left(Q, H_{t}\right)$-martingales. As a consequence,

$$
A=E^{Q}\left[A \mid H_{t}\right]+\sum_{i=1}^{n} \int_{t}^{T} \theta_{s}^{i}\left(d N_{s}^{i}-\alpha_{s}^{i} d s\right) .
$$

The latter expression can be interpreted in the following way. Starting from $t$, one can replicate the payoff $A$ with the initial investment $V_{t}=E^{Q}\left[e^{-r(T-t)} A \mid H_{t}\right]$ (in the savings account) and the trading strategy based on instantaneous digital credit default swaps defined by $\delta_{s}^{i}=\theta_{s}^{i} e^{-r(T-s)}$ for $0 \leq s \leq T$ and $i=1, \ldots, n$. As there is no charge to enter an instantaneous digital credit default swap, $V_{t}=E^{Q}\left[e^{-r(T-t)} A \mid H_{t}\right]$ corresponds to the time- $t$ replication price of the claim $A$.

Let us remark that it is theoretically possible to build up a replication strategy based on actually traded credit default swaps. Thanks to the predictable representation theorem, one can express the dynamics of credit default swaps in terms of the dynamics of instantaneous digital CDS. The next step consists on inverting a linear system to obtain the representation of any $H_{T}$-measurable payoff with respect to the dynamics of actually traded CDS. The reader is referred to the first chapter of Cousin et al. (2009) for a thorough presentation of this method in the case of two names. Interestingly, as explained in Cousin and Jeanblanc (2010), the dynamics of portfolio loss derivatives can be fully described using the dynamics of the underlying CDS when default times are assumed to be ordered. In this particular case, the hedging strategies can be computed explicitly in a general $n$-dimensional framework.

\footnotetext{
${ }^{12}$ or albeit $H_{T}$-measurable.
} 
While the use of the representation theorem guarantees that, in our framework, any basket default swap can be perfectly hedged with respect to default risks, it does not provide a practical way of constructing hedging strategies. As this is the case with interest rate or equity derivatives, exhibiting hedging strategies involves some Markovian assumptions.

\section{I.7 Computation of hedging strategies in a homogeneous Markovian setting}

When going to implementing actual hedging strategies, one needs extra assumptions, both for the implementation to be feasible and to cope with quoted CDO tranches. We therefore consider the simplest way to specialize the above model: we assume that all risk-neutral predefault intensities are equal and only depend on the current number of defaults, i.e.,

$$
\alpha_{t}^{i}=\tilde{\alpha}\left(t, N_{t}\right) 1_{\left\{t<\tau_{i}\right\}}, i=1, \ldots, n,
$$

where $N_{t}=\sum_{i=1}^{n} N_{t}^{i}$ denotes the total number of defaults that have occurred in the portfolio up to time $t$. We also assume that all recovery rates are constant across names and time. As a consequence, the loss process is merely proportional to the number of defaults process $N_{t}$ whose intensity is simply equal to the pre-default intensity times the number of non-defaulted names:

$$
\lambda\left(t, N_{t}\right)=\left(n-N_{t}\right) \tilde{\alpha}\left(t, N_{t}\right) .
$$

In that framework, it can be shown that the aggregate loss process is a continuous-time Markov chain, more precisely a pure death process (thanks to the no simultaneous defaults assumption) with generator matrix:

$$
\Lambda(t)=\left(\begin{array}{ccccc}
-\lambda(t, 0) & \lambda(t, 0) & 0 & 0 & 0 \\
0 & -\lambda(t, 1) & \lambda(t, 1) & 0 & 0 \\
& & \ddots & \ddots & \\
0 & 0 & 0 & -\lambda(t, n-1) & \lambda(t, n-1) \\
0 & 0 & 0 & 0 & 0
\end{array}\right) .
$$

Moreover, the replication price of a European-type CDO tranche payoff $\Phi\left(N_{T}\right)$ can be written as $V(t, k)=E\left[e^{-r(T-t)} \Phi\left(N_{T}\right) \mid N_{t}=k\right]$ and solves the backward Kolmogorov differential equations:

$$
\frac{\partial V(t, k)}{\partial t}=r V(t, k)-\lambda(t, k)(V(t, k+1)-V(t, k)), k=0, \ldots, n-1 .
$$

Regarding the hedging issue, homogeneous Markovian models are appealing because replication strategies are the same for all (the non-defaulted) names, which results in a dramatic dimensionality reduction. In that case, it is enough to consider the index portfolio as a single hedging instrument, which is consistent with some market practices. If we denote by $V^{I}\left(t, N_{t}\right)=E\left[e^{-r(T-t)} \Phi^{I}\left(N_{T}\right) \mid N_{t}\right]$ the time- $t$ replication price of the CDS index (Europeantype payoff), then by standard Itô's calculus, one can show that

$$
d V\left(t, N_{t}\right)=\delta^{I}\left(t, N_{t}\right) d V^{I}\left(t, N_{t}\right)+\left(V\left(t, N_{t}\right)-\delta^{I}\left(t, N_{t}\right) V^{I}\left(t, N_{t}\right)\right) r d t
$$

where: 


$$
\delta^{I}\left(t, N_{t}\right)=\frac{V\left(t, N_{t}+1\right)-V\left(t, N_{t}\right)}{V^{I}\left(t, N_{t}+1\right)-V^{I}\left(t, N_{t}\right)}
$$

is the credit delta, i.e., the proportion of the self-financing hedging portfolio invested in the CDS index. In other words, the change in value of a CDO tranche in a short period $[t, t+d t]$ can be fully replicated by holding at time $t$ a position $\delta^{I}\left(t, N_{t}\right)$ in the CDS index, for a total value of $\delta^{I}\left(t, N_{t}\right) V^{I}\left(t, N_{t}\right)$, and by investing the remaining part of the portfolio value, $V\left(t, N_{t}\right)-\delta^{I}\left(t, N_{t}\right) V^{I}\left(t, N_{t}\right)$, in the risk-free asset. The numerical implementation of hedging strategies can be achieved in a more realistic case through a binomial tree as detailed in Laurent et al. (2007) or by means of Markov chain techniques.

Eventually, we have build up a complete market model in which CDO tranche prices can be fully replicated by dynamically trading the CDS index and the risk-free asset and the associated hedging strategies can be derived explicitly. On practical grounds, another nice feature of the model concerns the estimation of model parameters from CDO tranche market quotes. As described in Laurent et al. (2007), the knowledge of upfront premiums of equity CDO tranches with different maturities and detachment points (and given some recovery rate) is equivalent to the knowledge of marginal distributions of the number of defaults at different time horizons. Thanks to the forward Kolmogorov equations, one can then perfectly compute the intensities of the aggregate loss process or the pre-default intensities. Such fully calibrated and Markov model is also known as the local intensity model. This parallels the local volatility approach of Dupire (1994) in the equity derivatives context where the dynamics of underlying assets are driven by a diffusion process as opposed to a finite state Markov chain in the case of credit portfolio derivatives. As in local volatility models, local intensity models allow for a perfect match of unknown parameters from a complete set of CDO tranches quotes. In this context, Cont et al. (2009) have computed an analogue, for credit portfolio derivatives, of the Dupire's well known formula. This is based however on the (rather hidden) assumption of no simultaneous defaults.

Another promising approach regarding hedging in a Markovian environment is the Markov Copula approach developed by Bielecki et al. (2008). In this full dynamic bottom-up framework, default indicators form a multivariate Markov process. The important point is that each individual default indicator is assumed to be a Markov process (in its own filtration). Under the latter assumption, there is no contagion effect in the sense that the default of a given name does not yield a change in the default intensities of the non-defaulted names. However, as this is also the case in common-shocks models, defaults may occur simultaneously ${ }^{13}$. The latter assumption is crucial on practical grounds since, it allows the calibration of model parameters to be dealt with exactly the same way as in a standard static copula set-up. Indeed, the calibration process can be performed in two separate steps. Firstly, individual default intensities can be pre-calibrated on single-name CDS curves and secondly, dependence parameters (intensities of joint defaults) can be fitted on CDO tranche quotes. Bielecki et al. (2010) provide a common-shocks model interpretation of this framework so that efficient convolution recursion procedures are also available for pricing and hedging static basket instruments like CDO tranches. Additionally, the Markovian structure of the model allows one to address the hedging issue in a dynamic and theoretically consistent way.

\footnotetext{
${ }^{13}$ The possibility of simultaneous defaults may also be seen as an extreme contagion effect.
} 
Until now, we have considered that credit spreads are driven by defaults and we have stressed that it leads to a complete CDO tranche market where the hedging can be fully described in a dynamical way. We will now use the same methodology to analyze the hedging issue in a completely different framework where spreads and defaults are both driven by a multivariate diffusion process. This corresponds to a multivariate extension of the Black-Cox (1976) structural model.

\section{I.8 Hedging credit portfolio derivatives in multivariate structural models}

One may compare the previous framework with the standard structural approach, where default time of a given name is defined as the first hitting time of a barrier by a geometric Brownian motion associated with the asset process of the corresponding name. Hull et al. (2005) investigate the pricing of CDO tranches within a Gaussian multivariate structural model, similar to the one presented in this section. Let $\left(W_{t}\right)_{t \geq 0}, W_{t}=\left(W_{t}^{1}, \ldots, W_{t}^{n}\right)$ be a $n$ dimensional Brownian motion whose components are correlated by the same dependence parameter $\rho$, i.e., for any $i \neq j,\left\langle W^{i}, W^{j}\right\rangle_{t}=\rho t$. We denote by $\left(F_{t}^{i}\right)_{t \geq 0}$, the natural filtration associated with $W^{i}, i=1, \ldots, n$ and $\left(F_{t}\right)_{t \geq 0}, F_{t}=\underset{i=1}{\vee} F_{t}^{i}$ the natural filtration associated with $W$. For any firm $i=1, \ldots, n$, we consider that the asset value $A^{i}$ follows a non-negative diffusion process under the historical probability measure $P$, i.e.,

$$
\frac{d A_{t}^{i}}{A_{t}^{i}}=\mu_{i} d t+\sigma_{i} d W_{t}^{i}, i=1, \ldots, n,
$$

where the expected rate of return $\mu=\left(\mu_{i}\right)_{1 \leq i \leq n}$ and the diffusion rate $\sigma=\left(\sigma_{i}\right)_{0 \leq i \leq n}$ are $\mathbb{R}^{n}$ valued. In this framework, the default times are defined by $\tau_{i}=\inf \left\{t \geq 0 \mid A_{t}^{i} \leq b_{i}\right\}$, where $b_{i}$ denotes the threshold associated with firm $i$. In that structural model, dependence between default times stems from the correlation between the asset values. For calibration of default probabilities over different time horizons, the barrier needs however to be time dependent, which does not change the main features of the model.

Roughly speaking, the value of assets for a given firm $i$ can be replicated by holding the debt and the equity part of its liabilities which can both be seen as tradable securities. This legitimates, at least on theoretical grounds, the role of firm's assets as primary hedging instruments in this framework. Using a multivariate extension of the Girsanov's theorem for correlated Brownian motion, it is possible to define a pricing measure $Q$, equivalent to the historical probability measure $P$, and such that discounted asset values are $Q$-martingales. Moreover, the latter change of probability measure does not perturb the dependence structure among dynamics of asset values. More specifically, the new Brownian motions $\tilde{W}^{1}, \ldots, \tilde{W}^{n}$ driving the dynamics of $A^{1}, \ldots, A^{n}$ under the probability $Q$ are still correlated with the same dependence parameter $\rho$, i.e., for any $i \neq j,\left\langle\tilde{W}^{i}, \tilde{W}^{j}\right\rangle_{t}=\rho t$. Furthermore, it is well known that the completeness of the market is guaranteed in this framework as far as the correlation matrix of $W$ involving parameters $\sigma_{1}, \ldots, \sigma_{n}$ and $\rho$ is invertible. One can then perfectly replicate any $F_{T}$-measurable payoff by holding a self-financing portfolio composed of firm's assets $A^{1}, \ldots, A^{n}$ and the risk-free asset. 
In this first-passage structural model, the cash-flows of credit default swaps written on name $i=1, \ldots, n$ can be synthesized as a combination of barrier options. Indeed, the cash-flows on the default leg are the same as the ones of a down-and-in barrier option with a fixed loss payment at the time when the value of assets falls below the pre-specified threshold. As for the premium leg, it can be replicated using a set of down-and-out barrier options with maturity dates equal to premium payment dates. It is then possible to relate the replicating price of a credit default swaps to replicating prices of barrier options. Clearly, the price of a CDS written on a given name $i$ is a Markov process with respect to the natural filtration associated with $\tilde{W}^{i}$ since the cash-flows are only driven by the dynamics of $\tilde{W}^{i}$. As a result, the dynamics of CDS $i$ only involves the dynamics of $\tilde{W}^{i}$.

Credit default swaps are described here as derivative instruments, but they can be used to dynamically hedge more complex products such as CDO tranches. This relies on the building of a self-financing portfolio including the risk-free asset and the dividend-bearing credit default swaps. Once the dynamics of each individual CDS has been found, it is straightforward to describe the dynamics of the replicating portfolio, given some pre-specified hedging strategies (predictable processes).

The hedging of CDO tranche price is then theoretically feasible thanks to the predictable representation theorem for multivariate Brownian martingales that holds under the pricing measure $Q^{14}$. Let us consider some $F_{T}$-measurable $Q$-integrable payoff $A$. This typically includes the payoff of CDO tranches or basket default swaps ${ }^{15}$ maturing at date $T$. Then there exists some $F_{t}$-predictable processes $\theta^{1}, \ldots, \theta^{n}$ such that:

$$
A=E^{Q}\left[A \mid F_{t}\right]+\sum_{j=1}^{n} \int_{t}^{T} \theta_{s}^{j} d \tilde{W}_{s}^{j} .
$$

Hence, the dynamics of CDO tranche prices can be described in terms of the dynamics of the correlated Brownian motions $\tilde{W}^{1}, \ldots, \tilde{W}^{n}$. Theoretically, the hedging strategies can be found by identifying the Brownian terms in the dynamics of the replicating portfolio and in the dynamics of CDO tranche prices ${ }^{16}$.

Let us note that when a default event occurs, the hedging position in the corresponding CDS is used to cover the loss. Then, from that time, the hedging portfolio contains one fewer CDS than before. But, since future cash-flows of CDO tranches are driven by the possible defaults of the non-defaulted names, CDS are not anymore required for hedging after extinction. This feature should be captured by the hedging strategies computed in this framework.

\footnotetext{
${ }^{14}$ Let us note that this theorem is usually written for martingales adapted to a filtration generated by a standard Brownian motion with independent components. However, as an intermediary step, the correlated Brownian motion $\tilde{W}$ can be expressed as the product of the square root of its correlation matrix with a standard $n$ dimensional Brownian motion with independent components. As a result, any payoff contingent to the dynamics of $\tilde{W}$ up to time $T$ can be described as a sum of stochastic integrals with respect to $\tilde{W}^{1}, \ldots, \tilde{W}^{n}$.

${ }^{15}$ At least when recovery rates are assumed to be deterministic.

${ }^{16}$ Let us note however that the dynamics of CDO tranche prices is very difficult to make explicit in this framework since it involves the joint law of some correlated first passage times.
} 
On practical grounds, one can remark that prices of credit default swaps and CDO tranches are Markovian processes in this framework and, by Feynman-Kac's theorem, they are also solutions of particular partial differential equations (PDE) ${ }^{17}$.

While the former Markov chain approach focused on default risk, neglecting credit spread risk, the structural approach deals with credit spread risk only. Default times are predictable stopping times with respect to the Brownian filtration and do not constitute an extra source of risk. Finally, the structural approach defines a complete CDO tranche market governed by spread risk and in that sense it can be seen as the dual counterpart of the Markovian contagion approach.

Fermanian and Vigneron (2009) tackle the problem from a slightly different perspective. Starting from a one factor Gaussian copula model, they are able to specify the dynamics of zero-coupon CDS (or equivalently conditional survival probabilities) that leads to perfect replication of European-type payoffs. Unsurprisingly, the completeness of the market is guaranteed when the correlation parameter used in the one factor Gaussian copula model corresponds to the correlation between Brownian motion driving the dynamics of zero-coupon CDS. Subsequently, they attempt to relate their framework with the multivariate extension of the structural model presented above but numerical investigation in that case seems to be cumbersome and is postponed for further research. Using market quotes of CDO tranches on recent series of iTraxx and CDX main indices, they instead perform a back-test investigation of correlation parameters (called break-even correlation) that would have been plugged in the one-factor Gaussian copula model in order to perfectly replicate a CDO tranche with the underlying single-name CDS, given a spread scenario. This method is a first step towards the practical implementation of hedging strategies in a complete market model driven by credit spread risks.

Multivariate structural models are consistent with popular dynamic credit risk models such as CreditMetrics ${ }^{\mathrm{TM}}$ and Moody's KMV. They permit to deal with joint credit migration (changes of credit ratings) amongst a wide range of names and with stochastic recovery, following the lines of Krekel (2008). Clearly, the multivariate Gaussian assumption is simplistic and questionable, but as in the previous Markov chain allows to derive unambiguous selffinancing replicating strategies for CDO tranches.

\section{I.9 Comparison with other approaches}

We have presented a dual view of complete market models, the former focusing on default risks while the latter concentrates on credit spread risks. The nature of default times differs from one case to another. In the former, default times are totally inaccessible stopping times, while in the latter case, default times are predictable. Given these two scholar models, we will set out the main theoretical features of hedging CDO tranches, coming to light whatever the underlying model. We will also try to highlight the commonalities (if any) between the approaches.

\section{a) Filtration}

\footnotetext{
${ }^{17}$ In the case of CDS, these PDE can be solved numerically either using finite difference methods or algorithms based on recombining trees. Regarding CDO tranche prices, the dimension of the PDE is too large to consider a direct numerical resolution method. CDO tranche prices should be computed using Monte Carlo simulations instead.
} 
A first issue is related to the choice of filtration. In order to deal with the hedging issue in a consistent dynamical way, one must start with the specification of a filtration. However, there are several ways of defining the information flow that is available to the modeller.

\section{Enlargement of filtration}

A first approach (generally associated with reduced form models) involves a reference or background filtration, generally driven by a jump-diffusion process which captures the evolution of some macro-economic factors or prices of default-free assets. An important point is that the background filtration does not provide enough information to predict with certainty at a given date whether the default of a name occurs or not. In other words, default times are not stopping times with respect to the background filtration. One needs some extra information to predict defaults. A natural idea is to expand this initial filtration with the flow of information provided by the dynamics of defaults. This is referred to as the progressive enlargement of filtration technique which has been extensively studied in the literature. An important issue associated with this technique is the so-called immersion property or $\mathrm{H}$ hypothesis under which martingales in the reference filtration remain martingales in the global enlarged filtration. Indeed, to preclude arbitrage opportunities in the default-free market, discounted default-free asset prices are assumed to be martingales with respect to the reference filtration (under a risk-neutral measure). Under the H-hypothesis, the no-arbitrage property is preserved in the global filtration, which is only the case in very particular situations. This has been pointed out by Jeanblanc and Le Cam (2009) in a single-name setting or by Bielecki et al. (2010) for top-down credit risk models. For instance, it is well known that the $\mathrm{H}$-hypothesis is satisfied in a single-name Cox model where the default time corresponds to the first jump instant of a doubly stochastic Poisson process. This is also the case in some very particular multivariate extensions of the latter framework where default times are assumed to be independent or ordered as shown in Ehlers and Schönbucher (2009).

\section{Direct specification of the entire set of information}

Another interesting and promising approach is the one proposed by Hitier and Hubert (2009). Compared with the previous approach, this construction follows the opposite direction. Hitier and Hubert (2009) consider a filtration for which default times are totally inaccessible stopping times and admit default intensities adapted to this filtration. Using the terminology of Jeanblanc and Le Cam (2007), this corresponds to an "intensity based approach" as opposed to the previous enlargement of filtration approach also called "hazard process approach". The idea is then to divide the initial filtration into two subfiltrations, the first being associated with a background intensity process independent of default events and the second being driven by defaults. They however assume that the background intensities are equal to the initial intensities before the arrival of the corresponding default and show that this hypothesis is implied by the classical H-hypothesis. An alternative route (generally associated with structural models) is to start with a filtration under which default times are predictable stopping times (or at least accessible, i.e., not totally inaccessible). This is typically the case for jump-diffusion structural models for which the initial filtration contains information associated with the asset value process and possibly related to a random barrier.

\section{Alteration or reduction of the initial set of information}

However, it is reasonable to assume that CDS or bond investors do not have the same information set as firm managers. Starting from a structural approach, there are several ways 
of specifying how the information set can be altered or obscured. Duffie and Lando (2001) build up the investor's filtration by first adding noise to the asset value process and sampling this noisy information set at some particular discrete dates. Goldberg and Giesecke (2004) start with a random-barrier structural model and assume that the threshold level is not known by the investor. Çetin et al. (2004) assume that the investor only observe whether the firm's cash-flows are positive or negative. In the approach by Guo et al. (2005), the asset value is only available at some discrete dates and with an additional delay or lag. In all the latter approaches, it is assumed that the investor can also observe the default event. As a consequence, the set of information available to the investor is defined by the smallest filtration containing the set of altered information and such that default times are stopping times. Interestingly, under this coarser filtration, default times are totally inaccessible stopping times and admit default intensities yielding to a reduced form modelling approach. As we fall in the class of reduced form approach, a natural investigation is to check whether the immersion property holds. When the asset value process is obscured by a diffusion process, this is effectively the case as shown in Coculescu et al. (2008). The reader is referred to Jarrow and Protter (2004) for a detailed analysis of the link between structural and reduced form models in the case of incomplete information.

Whatever the techniques used to specify how information is dynamically disclosed to the modeller, default times are finally always defined as stopping times with respect to a given filtration. However, depending on the modeller's access to the overall information, default times can be either predictable, totally inaccessible stopping times but also stopping times which are neither predictable nor totally inaccessible (as this is the case for instance for jumpdiffusion structural models where negative jumps can impact firms assets' dynamics).

\section{b) Martingale representation theorem and completeness of the market}

The existence of a martingale representation theorem is a key ingredient regarding the hedging issue. Given a model specification, i.e., introduction of an underlying probability measure, description of sources of randomness, and construction of an information set, it provides a nice representation of martingale processes in terms of fundamental elementary martingales such as Brownian motions or compensated (marked) point processes. More precisely, it states that the dynamics of any martingale process can be expressed as a linear combination of some fundamental martingales, the weights being associated with some predictable processes. In particular, it allows one to represent the dynamics of any contingent claim in terms of the dynamics of the fundamental martingales. The martingale representation theorem is thus the right mathematical tool to study the completeness of the market model.

\section{c) Hedging instruments}

Another common ingredient associated with the hedging issue concerns the specification of tradable hedging instruments. Let us note obviously, that the question of the market completeness has to be addressed in connection with both the specification of a market model and the description of some liquid hedging instruments. Typically, one could think of credit index default swaps, CDS on names with possibly different maturities, standardized synthetic single tranche CDOs. However, to simplify the theoretical analysis, it is often convenient to assume in a first stage that stylized default-contingent products are traded on the market, provided that actually traded hedging instruments can be fully replicated using these simplified products. 


\section{e) Change of probability measure}

The natural way of defining a credit risk model is to start with the construction of default times under the physical probability measure. However, when arbitrage opportunities are precluded, one can find an equivalent probability measure also called pricing or risk-neutral measure under which discounted prices of (non-dividend bearing) primary assets are martingales. This is very convenient both on practical and theoretical grounds. The pricing measure is built up thanks to an equivalent change of probability measure involving a RadonNikodym density similar to the one involved in the classical Girsanov's theorem. However, Coculescu et al. (2007) show that, in an intensity model, the immersion property is not preserved by an equivalent change of probability measure, which may explain why in so many approaches, the model is directly specified under a given risk-neutral measure.

\section{II) From theory to hedging effectiveness}

\section{In theory, theory and practice are the same. In practice, they are not. Albert Einstein}

Starting from the simplest cases of a complete market and either Markov chain or structural models, we tried to show that most multivariate models of defaults involve more or less the same basis ingredients, though we still lack a simple to state and comprehensive mathematical framework.

Rather than concentrating on the internal features of a pricing (and possibly hedging) model, such as the relevant filtrations, conditional default probabilities, aggregate loss or credit spread dynamics, we should think of the outcomes of a hedging model for CDO tranches. These are rather simple: a set of hedging instruments, CDS in most cases, sometimes other CDO tranches are also considered, and hedge ratios with respect to the hedging instruments. A key point is that the outputs are the same for instance in the structural or in the Markov chain models described above. Thus, it will be possible to derive some comparisons between models built upon different premises, for instance their ability to reduce the risk of a CDO tranche through a dynamic trading strategy. This will lead to back-testing approaches and it turns out to be an econometric rather than a theoretical issue.

More precisely, a pricing and hedging model is usually associated with a parameterized set of trading strategies and to proceed with any comparison, we must specify how the parameters, for instance some correlation or contagion parameters, are set and updated. As will be discussed below, such a choice is likely of first importance with respect to hedging performance, though such calibration or estimation of hedging parameters is usually neglected in theoretical approaches.

We do not claim that pricing is irrelevant with respect to the hedging problem. Usually, pricing models are calibrated on liquid market quotes ${ }^{18}$. Then, such a calibrated model can be used in a predictive way. If we assume that the implied parameters, say, correlation parameters for base tranches, are constant over some (short) time interval or evolve according to some predefined rule, then, given some new quotes of underlying CDS (say), one should be able to predict the change in market value of a tranche and thus the required amount of hedging CDS. Thus, pricing models that are associated with implied parameters with smooth patterns over time are likely to be useful for risk management purpose too. The same line of

\footnotetext{
${ }^{18}$ Provided that these are available, an assumption which is problematic during periods of market disruption.
} 
reasoning will be detailed below when considering the connection between hedging and the pricing of bespoke CDO tranches.

Going further along these general statements, we also recall that any pricing and hedging model associated with perfect replication will be rejected by data with probability one: first, such models are associated with non noisy relationships between CDO tranches. Then, completeness precludes any hedging residual. We thus deal with misspecified models and should not consider such misspecification as a rejection a priori of a pricing and risk management framework. We should rather focus on the degree of misspecification as for instance assessed by hedging performance ${ }^{19}$.

Another important practical topic is related to stability of hedging performance over different periods and under different economic regimes or market contexts. Certainly, one would most likely chose an all-roader that could both cope with the idiosyncratic auto crisis of 2005 and the systemic crisis of 2007-2009. This view is also related to a stationary world and objective probabilities, which is rather comfortable, but is also questionable when dealing with financial markets. The notion of regimes of volatility, which has been popularized by Derman (1999) in equity markets might also be applicable in correlation markets. Clearly, identifying the relevant regime is an issue, but it seems a rather unrealistic objective that a simple to implement hedging strategy would supersede its competitors in any context $\mathrm{t}^{20}$.

The above remarks may look thought-provocative from the standard mathematical finance and modelling point of view. We do not claim that a model does not need to rely on understandable, sound and clear economic intuition and internal consistency. But on the other hand, looking for the perfect fit leads to extra implementation costs, blurs the key and ancillary features, obscure internal and external communication about risk management and increase operational risks.

This is why we deliberately favoured the multivariate structural model and the Markovian loss models ${ }^{21}$. Both allow the derivation of replicating strategies of CDO tranches. Calibration is, at least at first sight, rather easy. Moreover, the one factor Gaussian copula has become the standard model as measured by market acceptance, for pricing and hedging purpose (see Finger (2009)). From Hull, Predescu and White (2005) or Cousin and Laurent (2008a), we know that the Gaussian copula dependence is quite similar to the one that comes out of the multivariate structural model: At least for investment grade names, default is not likely to occur shortly after inception. The Gaussian copula might be viewed as a one step version of the multivariate structural model. Thus, deltas with respect to underlying CDS are likely to be quite similar in the two approaches. We will follow thereafter this route ${ }^{22}$. Since deltas

\footnotetext{
${ }^{19}$ One could argue that the new generation of top-down models takes the loss surface or equivalently the set of CDO tranches quotes as inputs, as is the case with HJM models in the interest rates framework. However, such models are not aimed at risk managing plain CDOs, which seems to be the key issue today, but rather focus on more complex payoffs, such as CPDOs or leverage super senior tranches.

${ }^{20}$ In a recent work, Cont et al. (2009) investigate the hedging abilities of the Gaussian copula model and of the Markovian contagion model (local intensity model in their terminology). They look for regimes where one model would outperform the other. They show some robustness of the Gaussian copula approach.

${ }^{21}$ Frey and Backhaus (2007, 2008), Arnsdorf and Halperin (2007) provide some Markovian loss models which are more versatile, but also more difficult to handle.

${ }^{22}$ We might have alternatively considered the Gaussian copula as a (non Markovian) contagion model. If one restricts to the natural filtration of default times, defaults are informative; they bring some information about the latent Gaussian variables of the non defaulted names. The jumps of credit spreads at default times depend upon derivatives of the survival function and there is a singularity at time zero. This is not however the common way to appraise the Gaussian copula. If one were to observe the path of assets associated with different names instead
} 
associated with the multivariate structural model are well understood and lead to perfect replication of CDO tranches when concentrating on spread risk (defaults are predictable), we can think of deltas coming out of the Gaussian copula model to have some economic significance $^{23}$. As discussed above, Fermanian and Vigneron (2009) propose a different approach. They look for some dynamic models consistent with the credit spread deltas obtained from the Gaussian copula model.

\section{II.1 Hedging with CDO tranches}

If you want to know the value of a security, use the price of another security that is as similar to it as possible. All the rest is modeling. Emmanuel Derman

Hedging of CDO tranches with other tranches on similar underlying portfolios, with close attachment-detachment points and maturities is likely to be the more efficient and least costly way of pricing and risk managing a book. To go a bit further, we could speak of spatial versus dynamic hedging. To properly implement such a static hedging some kind of metrics or proximity between payoffs is required and this usually involves a model. For instance, given a joint distribution of default times in a bottom-up model, one might use a set of liquid CDO tranches to minimize the $\ell_{2}$ - norm hedging error within a book of bespoke tranches ${ }^{24}$.

One could either proceed with a linear regression based on historical data. Obviously, to go along this approach, historical data is required ${ }^{25}$. Let us for instance consider the hedging of an equity tranche with a junior mezzanine tranche on a standard index such as iTraxx Europe Main or CDX NA IG. Here, one has to keep in mind that the historical and the model based approaches to static hedging are rather different. There is no reason why the static hedge ratio, i.e., the coefficient in the regression of the payoff to be hedged on the hedging payoff has to be constant over time. For instance, going further in the above example, as the expected loss on the underlying portfolios changes over time, the moneyness of the equity and junior mezzanine tranches do not change accordingly and the ratio of theoretical deltas (in almost any theoretical hedging model) is likely to vary. This first point is fairly obvious. Also, at different points in times, the market views may change. This does not concern only the mean of the underlying portfolio, but other statistical features, such as, say, the degree of dependence between default times. Eventually, there is no reason of a stable and linear relationship between tranches over time which weakens the purely statistical approach and calls for the use of a model for conditional (upon the current information) and epistemic ${ }^{26}$ distributions of default times.

\footnotetext{
of the terminal values, contagion would disappear. About ten years ago, while one of the authors was working on basket credit derivatives, the preferred model was a structural model based on multidimensional NIG processes and a factor structure. Prices and Greeks were computed by Monte Carlo, which was quite slow, especially for the Greeks. At one point in time, the model was downgraded to a static one, but in most cases, prices and Greeks were quite similar. We would definitely need a more systematic investigation of this point.

${ }^{23}$ In theory, one should start with the computation of the derivatives of the value of the CDO tranche and of hedging CDS with respect to underlying state variables, here the asset values at the current time. Then, the amount of hedging CDS is the ratio of the above derivatives. Market practitioners rather make the derivation with respect to the default barrier, or in a static approach with respect to the default threshold. This can also be seen as a shift in credit spreads, often of one basis point.

${ }^{24}$ Given a conditional (upon current information) joint distribution of default times, it is possible to compute the covariances of the bespoke payoff and of the liquid tranche payoffs and thus to derive the optimal static hedge.

${ }^{25}$ Typically, daily or weekly data are used. This requires prices for the payoffs to be hedged and the hedging instruments. As a consequence, this approach is not well suited to hedge non standard tranches.

${ }^{26}$ Reflecting risk managers views about the future.
} 
Designing hedging strategies using CDO tranches does not require the minimization of some hedging error and can be conducted through some pricing model, usually some variant of the Gaussian copula model.

Based upon a simple implementation of the Gaussian copula model ${ }^{27}$, Ammann and Brommundt (2009) report that the standard deviation of the changes in value of a deltahedged tranche, with an adjacent tranche, is about two times smaller than that of an unhedged portfolio. One could expand upon that, and use the Gaussian copula model to hedge, say, a junior mezzanine tranche against an equity tranche and the credit default swap index. In theory, one should be able to hedge both against small and large movements of the index, that is both delta and (parallel) gamma risks. Actually, since equity tranches are associated with large idiosyncratic gamma risks, such approach is perilous. Some still remember that similar trades with positive carry were popular up-to the 2005 auto crisis, when some investors had to cut positions with heavy losses: see Petrelli et al. (2006, 2007). We can also mention that this kind of Taylor expansion based approach to hedging lacks some theoretical rigour since higher order terms may not be associated with vanishing risks. Given this, it is not surprising that hedging a given CDO tranches with two different tranches may lead to a decrease in hedging performance (Ammann and Brommundt (2009)).

An important and practical topic is the pricing and risk management of bespoke tranches. These are tranches based upon credit portfolios that differ from standard indexes, usually with higher credit spread names. Turc et al. (2006), Baheti and Morgan (2007) describe different ways to compute correlation parameters depending upon the average credit spread of the bespoke portfolio. Ding and Sherris (2009) use these ideas to build and check different hedging strategies of standard CDO tranches. Given some stated dependence between correlation and credit spreads, one can compute the total derivative of a CDO tranche with respect to a shift in credit spreads. For instance, using the moneyness matching approach in Turc et al. (2006), an increase in credit spreads is associated with an increase in the expected loss and thus a decrease in the detachment point of the "equivalent" equity tranche. Since base correlations curves are usually upward sloping, an increase in credit spreads is thus associated with a decrease in base correlations. Of course, keeping the correlation parameters constant is a special case corresponding to usual delta computations.

Another common issue is the hedging of non standard tranches on standard indices, such as iTraxx or CDX. It may also concern super-senior tranches or on the contrary first losses tranches, thin tranchelets, for instance a [5\%-6\%] tranche on the iTraxx and eventually short term contracts especially as older series mature. Some commonly described approaches lead to difficulties ${ }^{28}$. One has to deal with standard no arbitrage constraints on expected tranche losses, which are also related to super-replication prices as discussed in Walker (2008). This actually provides some help, but still leads to a wide range of admissible prices and hedge ratios in a number of cases. This also raises the issue of the availability and reliability of prices of such non standard tranches. During the liquidity and credit crisis, the spreads of super senior tranches widened dramatically. This was partly due to the fear of systemic and contagion effects in the credit world. But it may also be that huge short positions by major participants, such as AIG, could not be held due to the lack of collateral, and possibly some

\footnotetext{
${ }^{27}$ Large homogeneous portfolio approximation.

${ }^{28}$ For instance, careless approaches based upon the interpolation of base correlations may lead to negative tranchelets prices, the use of base correlations is associated with non local effects in correlation analysis and extrapolation is often hazardous. Moreover, hedge ratios also depend upon somehow arbitrary recovery rate assumptions. See below about delta scattering and model implementation.
} 
predatory trading have emphasized the price of super senior protection. In such a period of stress and market dislocation, one can rightfully wonder about the significance of transaction prices.

\section{II.2 Back to back-testing}

A theory is something nobody believes, except the person who made it. An experiment is something everybody believes, except the person who made it. Albert Einstein.

Let us now go back to comparing dynamic hedging strategies of CDO tranches based upon credit default swaps. We will focus upon a number of methodological issues and about some results that come out of the small number of empirical studies.

Our message is twofold:

First, there are numerous statistical and practical issues, when assessing hedging performance, which cannot clearly be seen from the bird's-eye view of academic mathematical finance. These will be detailed thereafter and as a consequence drawing comparisons between different "models" is difficult.

Then, credit spread deltas of CDO tranches, with respect to credit default swap indexes, as obtained from the Gaussian copula approximation of the structural model and default deltas are not that different one from another. Moreover, static deltas obtained from dynamic Cox models are not different either. The discrepancies between the models can be given some economic intuition, based upon the correlation dynamics or equivalently the mechanism of default contagion. These deltas lead to risk reducing hedging strategies. Actually, it suggests that market practice is far from being vacuous, since risk management amongst trading desks relies mainly upon variations of the Gaussian copula approach. This is rather good news at the level of an investment bank, though it does not necessarily solve for systemic issues, where major trading firms appear to have similar long or short exposures to be hedged.

Our main focus regarding comparing models will be the use of the Gaussian copula and of the Markov loss model described above. As discussed earlier, the Gaussian copula is viewed here as some approximation of a costly to implement structural model. Both models are widely used amongst practitioners and have sound and easy to understand theoretical foundations. We will not pay of lot of attention to so-called intensity models, one should either say models based on Cox processes, due to their difficulties in dealing with the high degree of dependence between default events, as seen during the credit and liquidity crisis ${ }^{29}$. Similarly, we will only briefly deal with incomplete market approaches even though some seem to be associated with appealing hedging efficiency. These approaches are a bit trickier on theoretical grounds, since the connection between the pricing and the hedging is not as obvious as in the case of complete market models.

Before going further in comparing hedging strategies, a few points need to be stated.

At this stage, partly due to practical constraints, back-test studies have involved the use of indexes rather than individual credit default swaps. In other words, the amount of credit default swap used in the hedging portfolio is name independent. This is questionable

\footnotetext{
${ }^{29}$ Regarding hedging issues, Laurent (2006) deals with such models. It is shown that due to default diversification in large portfolios, the hedging error can be controlled by hedging credit spread risk only.
} 
especially when the dispersion of credit spreads amongst the underlying portfolio is significant. For instance, such name heterogeneity is likely to involve higher credit deltas in equity tranches for risky names. This issue is discussed below where the scattering of individual name delta is being considered.

When trying to simultaneously deal with default and credit spread risk, a view shared by some academics is to deal with credit default swap indexes of different maturities. However, we still lack some empirical studies to support such a view and there might be some operational difficulties in implementing these ideas. For instance, hedge ratios could be of opposite signs and large magnitude. In the studies we are aware off, the preferred hedging instrument is thus the credit default swap index of the same maturity than the considered CDO tranche. It would definitely be some interesting investigation to assess whether the use of credit default swaps of different maturities actually increases hedging performance. Preliminary and indirect evidence discussed below tend to show that credit spread deltas and default deltas are not too far away and suggest that a given position in the underlying credit portfolio could lead at a good hedge both against default and credit spread risks.

Regarding the computation and the calibration of parameters, there are different issues ${ }^{30}$. We already mentioned that the Markovian loss model, in its simplest form, involves a set of contagion parameters which has the same dimension as the number of names (say 125), while there are much less pricing constraints (typically 6 for one horizon). Depending upon the calibration method, the dependence of the loss intensities to the number of defaults may vary. This is not innocuous from the point of view of delta computations.

In the Gaussian copula model, there are also a number of issues, related to the way correlation parameters are determined, whether base or implied correlations are being used, whether these correlations are kept constant or updated as credit spreads change (correlation regimes). The principles of the hedging and risk management are fairly simple: the pricing tools are used to compute sensitivities to market inputs and to market parameters, such as credit spreads of the constituents of the reference credit portfolio. The main focus is put on credit spread risk, while default risk is usually dealt with a reserve policy. Such risks are managed thanks to credit index default swaps or CDS on the underlying names of the basket. Other risks, such as idiosyncratic and parallel gamma ${ }^{31}$ credit spread risks, or correlation exposure can be in principle managed by trading liquid index tranches across the capital structure.

Let us emphasize a key issue when computing credit deltas in the one factor Gaussian copula model with base correlations. There are actually two approaches that can be denoted as "sticky strike" and "sticky delta" to parallel the terminology used in equity derivatives markets (see Derman (1999)). In the sticky strike approach, the base correlations are kept unchanged when bumping the credit curves. When computing "sticky deltas", one takes into account the change in base correlations due to the change in the moneyness of the tranche when credit spreads move up: the equity tranche becomes more junior, which actually leads to using a smaller base correlation. In other words, in the sticky delta approach an increase in credit spreads is associated with a smaller dependence between default events. As a consequence, the sticky delta of an equity tranche is lower than the delta computed under the sticky strike approach.

\footnotetext{
${ }^{30}$ We already briefly discussed some issues regarding the computation of hedge ratios when considering affine intensity models.

${ }^{31}$ Idiosyncratic Gamma is also denoted as iGamma and referred to as "microconvexity". Parallel Gamma is also known as Index Gamma and referred to as "macroconvexity".
} 
Morgan and Mortensen (2007) have investigated some anomalies when using the base correlation for the computation of sensitivities. They show that credit spread deltas on iTraxx S7 $5 \mathrm{Y}$ [12-22\%] tranche can be negative due to the steepness of the base correlation curve. Such a counterintuitive effect ${ }^{32}$ is illustrated in the graph below:

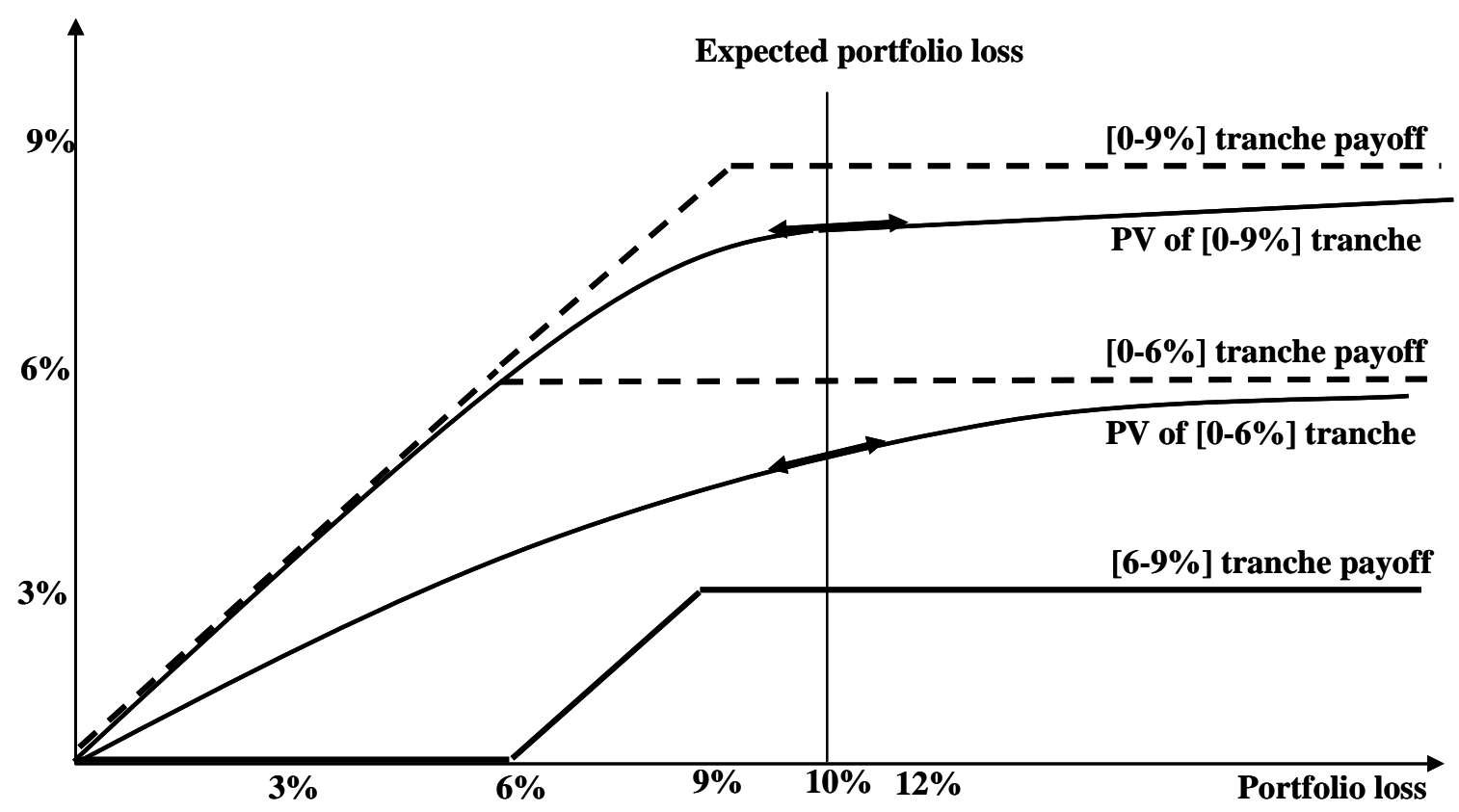

Figure 1. Negative deltas due to the steepness of the base correlation curve.

Example: consider a mezzanine [6-9\%] tranche on a bespoke portfolio. The expected loss on the portfolio at inception is equal to $10 \%$. Figures in solid lines show the present value of the default leg of the [0-6\%] and [0-9\%] base correlation tranches as a function of the expected loss on the portfolio. The base correlation for the [0-9\%] is assumed to be very high, thus the volatility of the reference portfolio is quite small as the time value of the base tranche. Conversely, the base correlation of the [0$6 \%$ ] tranche is much smaller which is consistent with steep upward base correlation curves. Thus, the volatility of the (same) reference portfolio is much higher as the time value of the option. The present value of the default leg of the mezzanine [6-9\%] tranche is the difference between the present values of the [0-6\%] and [0-9\%] base correlation tranches and should remain between 0 and $3 \%$ to avoid plain arbitrage opportunities. Given this constraint, it may be (see Figure 1) that the delta of the more junior tranche is smaller than the delta of the more senior tranche for some levels of expected portfolio loss. In such regions, the present value of the mezzanine tranche will decrease as the expected loss on the underlying portfolio increases which is rather unlikely.

Also, when considering a bump in credit spreads in order to compute a hedging exposure in the credit default swap index, one may, for instance, operate a translation or rather chose a multiplicative effect, which clearly will not lead to the same magnitude of credit deltas for the different tranches. Recently, recovery rate assumptions have appeared of key importance. Prior to the liquidity and credit crisis, most market participants relied upon a standard though arbitrary recovery rate assumption of $40 \%$. Since then, it appeared that such an assumption would not be consistent with the large spreads quoted on senior tranches. Various amendments, including a recovery markdown or different specifications of state dependent

\footnotetext{
${ }^{32}$ Schloegl et al. (2008) show that such effects can also occur in arbitrage-free models.
} 
stochastic recovery rate have been proposed by the industry (see Amraoui et al. (2009)). This has actually a significant impact upon hedge ratios, especially at the individual name level. Typically, the use of a stochastic recovery rate or a recovery rate markdown will tend to lower the exposure of senior tranches to tight names. The basis risk between the spread of the credit default swap index and the average spread of its constituents is another seemingly minor issue when computing hedge ratios. Such basis risk is mostly related to "transaction costs" and can fluctuate widely, especially during times of turmoil. This raises some doubts regarding the effective level of spreads and thus of expected loss and can have some effect on the computation of hedge ratios. Various choices and adjustments can be envisaged, none of them could actually be neglected when considering hedging efficiency. Other seemingly minor issues have to be dealt with in order to compute correlation parameters that are consistent with market quotes, such as the amortization of premium legs and the term structure of CDO tranche spreads ${ }^{33}$.

Let us stress that, when constructing hedge ratios, the use of parameters that calibrate prices may not be the first-best. Gouriéroux and Laurent (1996) have developed a concept of objective based inference and implied hedging parameter, which might be well suited when conducting back-tests with misspecified models.

Regarding hedging efficiency, we would first like to emphasize some similarities between the deltas associated with dissimilar models, Gaussian copula, Markov loss model and affine intensity models.

Eckner (2007) or Feldhütter (2008) rely on an affine specification of default intensities. Conditionally on the path of default intensities, default times are independent, i.e., there are no contagion effects at default times. The model is parametric with respect to the term structure of credit spreads and to CDO tranches. Eckner (2007) calibrates model parameters to credit spreads and liquid tranche quotes associated with the CDX NA IG5 index. Hedge ratios with respect to the credit default swap index are then computed ${ }^{34}$. The sensitivities of CDO tranche and index prices are calculated with respect to uniform and relative shifts of individual intensities. The model deltas can be compared with those computed from the Gaussian copula model. As can be seen from Table 1, though the figures differ, the orders of magnitude are roughly the same. The equity tranche deltas computed in Eckner (2007) are slightly larger than those computed under the Gaussian copula, as in a "sticky delta" approach. Such a result is consistent with a market where an increase in the average credit spread is the outcome of some idiosyncratic shifts and an increase in the dispersion of credit spreads. This is typical of the May 2005 correlation crisis, which was actually associated with smaller correlations on the equity tranches. At this stage, some methodological points are worth mentioning. First, while the model is dynamic, the way hedge ratios are computed is typically static. One shifts some parameters related to credit spreads without relating such bumps to a theoretical approach of dynamic hedging. More importantly, practical grounds, there are different ways to inflate credit spreads, associated with different hedge ratios. For

\footnotetext{
${ }^{33}$ We refer to Jobst (2007) or Meissner et al. (2008) (chapter 18 of Meissner (2008)) for further discussions about hedging CDOs within the Gaussian copula framework. On the numerical side, Andersen, Sidenius and Basu (2003), Iscoe and Kreinin (2007), among many other authors, provide semi-analytical techniques to compute sensitivities within the Gaussian copula framework. Joshi and Kainth (2004), Rott and Fries (2005), Chen and Glasserman (2008) detail some improvements of the Monte Carlo approach which are applicable to the pricing and hedging of CDO tranches, especially when one falls outside the factor framework.

${ }^{34}$ Let us remark that this model would hardly be calibrated during the 2008 crisis on CDX and iTraxx tranches.
} 
instance, one can privilege a shift associated with the systemic component of the intensity, such a shift will be therefore associated with an increase in the dependence between defaults.

\begin{tabular}{lccccc}
\hline Tranches & {$[\mathbf{0 - 3} \%]$} & {$[\mathbf{3 - 7} \%]$} & {$[\mathbf{7 - 1 0} \%]$} & {$[\mathbf{1 0 - 1 5 \% ]}$} & {$[\mathbf{1 5 - 3 0 \% ]}$} \\
\hline market deltas & 18.5 & 5.5 & 1.5 & 0.8 & 0.4 \\
model deltas & 21.7 & 6.0 & 1.1 & 0.4 & 0.1 \\
\hline
\end{tabular}

Table 1. Market deltas and model deltas as in Eckner (2007).

Arnsdorf and Halperin (2007) consider a Markov chain that accounts for the dynamics of defaults and credit spreads. This can be seen as a "two dimensional" Markov chain. Contrary to the previous model, defaults are informative and credit spreads jump at the arrival of defaults. The theoretical properties of the model with respect to completeness are not studied but Arnsdorf and Halperin (2007) compute deltas of standard iTrraxx tranches with respect to the corresponding credit default swap index. As in Eckner (2007), the deltas with respect to individual credit default swaps are not provided. However, one could think of using the random thinning procedure discussed in Giesecke and Goldberg (2005) or Giesecke (2008) to provide such individual deltas.

\begin{tabular}{lccccc}
\hline Tranches & {$[\mathbf{0 - 3} \%]$} & {$[\mathbf{3 - 6} \%]$} & {$[\mathbf{6 - 9} \%]$} & {$[\mathbf{9 - 1 2} \%]$} & {$[\mathbf{1 2 - 2 2 \% ]}$} \\
\hline market deltas & 26.5 & 4.5 & 1.3 & 0.7 & 0.3 \\
model deltas & 21.9 & 4.8 & 1.6 & 0.8 & 0.4 \\
\hline
\end{tabular}

Table 2. Market and model deltas as in Arnsdorf and Halperin (2007).

Table 2 shows some market (computed under the Gaussian copula model) and model deltas (corresponding to "model B" in Arnsdorf and Halperin (2007)) in March 2007, for five year CDO tranches. As in Table 1, it can be seen that the figures are roughly the same. However, it is noticeable that equity tranche deltas are smaller when using the Markov chain.

From a risk management perspective, an interesting feature is that the deltas with respect to underlying credit default swaps have the same order of magnitude in the different approaches. Let us first recall that, in the case of zero default-free interest rates, the default leg of a senior CDO tranche can be seen as a call option on a portfolio of discount bonds maturing with the CDO tranche. The above delta feature suggests that at a portfolio level, say an iTraxx or CDX index, a shift of credit spreads or a default event would have roughly the same effect on the expected portfolio loss dynamics. In other words, the same amount of CDS would lead to protection both against credit spread and default risks. This is quite preliminary and needs to be further confirmed by using an embedding framework.

Though the pricing methodology differs, Eckner (2007), Arnsdorf and Halperin (2007), Laurent et al. (2007), Cont and Kan (2008), Feldhütter (2008), Cont, Deguest and Kan (2009), Cont et al. (2009) provide some examples of the use of dynamic arbitrage free pricing models to compute sensitivities with respect to credit spreads and thus hedge ratios with respect to credit default swaps. As mentioned above driving comparisons is never an easy task since one has to decide about the way model parameters are determined. This can actually explain some discordance between empirical studies regarding hedging efficiency of different models, especially since there is not so much difference between the computed deltas. While, unsurprisingly, the authors stress the divergences between hedging strategies associated with different modelling approaches, the relative errors, as measured by root mean squared error or the mean average hedge error, do not differ that much. 
Regarding statistical issues, when assessing hedging performance, one should be cautious about the issues related to data snooping, multiple hypothesis testing or false discovery rate. It is tempting to embed a simple model in a larger one which is likely to be more flexible and involve more parameters. Then, the simple model appears to be restricted version of a more sophisticated one and we can check this restriction on parameters ${ }^{35}$. If the base model is not rejected, then most likely researchers will look for another extension until a new model is found which is claimed to supersede the older one. This is not a fair contest since the best alternative is chosen and one has to correct test statistics accordingly. In and out of sample do not correct for this data driven model selection, because comparing out of sample hedging performance is part of the standard model selection methodology. This strengthens our focus on simple models ${ }^{36}$.

\section{II.3 Delta scattering}

Up to now, we mainly concentrated on the hedging of CDO tranches with respect to the underlying credit default swap index. However, in many cases it makes sense to hedge at the name level, especially when the spreads associated with the index constituents are dispersed. A name with a large spread is more likely to contribute to the value of an equity tranche, and thus should be associated with a larger hedge ratio than a tight name. Likewise, an increase in dispersion of spreads should be associated with an increase in the value of the default leg of an equity tranche (and conversely for senior tranches).

By construction, bottom-up models and especially copula type models, allow a name per name derivation of hedge ratios ${ }^{37}$. Regarding top-down models, the building of individual deltas is currently being investigated; random thinning, as some researchers advocate, might do the job. We leave this point for further discussion.

Another issue with models than involve some kind of contagion is the scope of contagion effects. For instance, failure of a name within the CDX index may lead to jumps in the credit spreads of names in the iTraxx. In theory, such exposure should be hedged, which may be forgotten if one would consider the North American world separately. Likewise, such hidden name dependence appears in bespoke CDOs due to the mapping onto liquid indexes.

As mentioned in the first paragraph, one is likely to expect a smooth and increasing pattern of credit deltas with respect to the spreads of the underlying names for an equity tranche, a decreasing pattern for senior tranches and possibly a humped shape for some mezzanine tranches.

However, other issues came to light, such as huge discrepancies between individual name deltas and subsequently very large positive or negative idiosyncratic Gammas in high correlation regimes, as observed in 2008 on the iTraxx and CDX markets. Actually, when the dependence level between default dates becomes quite high, as could be seen during the

\footnotetext{
${ }^{35}$ This first step may not be straightforward since the distribution of hedging errors needs to be derived.

${ }^{36}$ Even though, looking in greater details, there are still a lot of rather arbitrary and often not noticed modelling choices.

${ }^{37}$ See Cousin and Laurent (2008a), Cousin and Laurent (2008c) or Burtschell et al. (2009) for reviews of such bottom-up models within a factor copula framework. The sensitivity approach applies to copulas models that, contrary to the base correlation approach, provide arbitrage-free CDO tranche quotes. Schloegl et al. (2008) prove that tranchelet sensitivities are always positive in such a framework. Dealing with large amounts of data and the cost of numerical implementation drove early back-test studies towards using credit default swap indexes.
} 
liquidity and credit crisis, strange phenomena occur. Names cluster together according to the level of credit spreads and credit deltas are either equal to zero or one (see Burtschell et al. (2009) for a detailed analysis). For instance, when looking at an equity tranche, the names with the highest credit spreads have a delta equal to one ${ }^{38}$, while the remaining names have a delta equal to zero. Such a phenomenon also occurs in the stochastic correlation model described by Burtschell et al. (2007). The bumps in Figure 2 are related to the comonotonic (perfect dependence) state and the heterogeneity amongst credit spreads. Such a rather counterintuitive pattern precludes the use of the credit default swap index as hedging tool for $\mathrm{CDO}$ tranches. Other heterogeneity effects in individual credit deltas are reported by Houdain (2006).

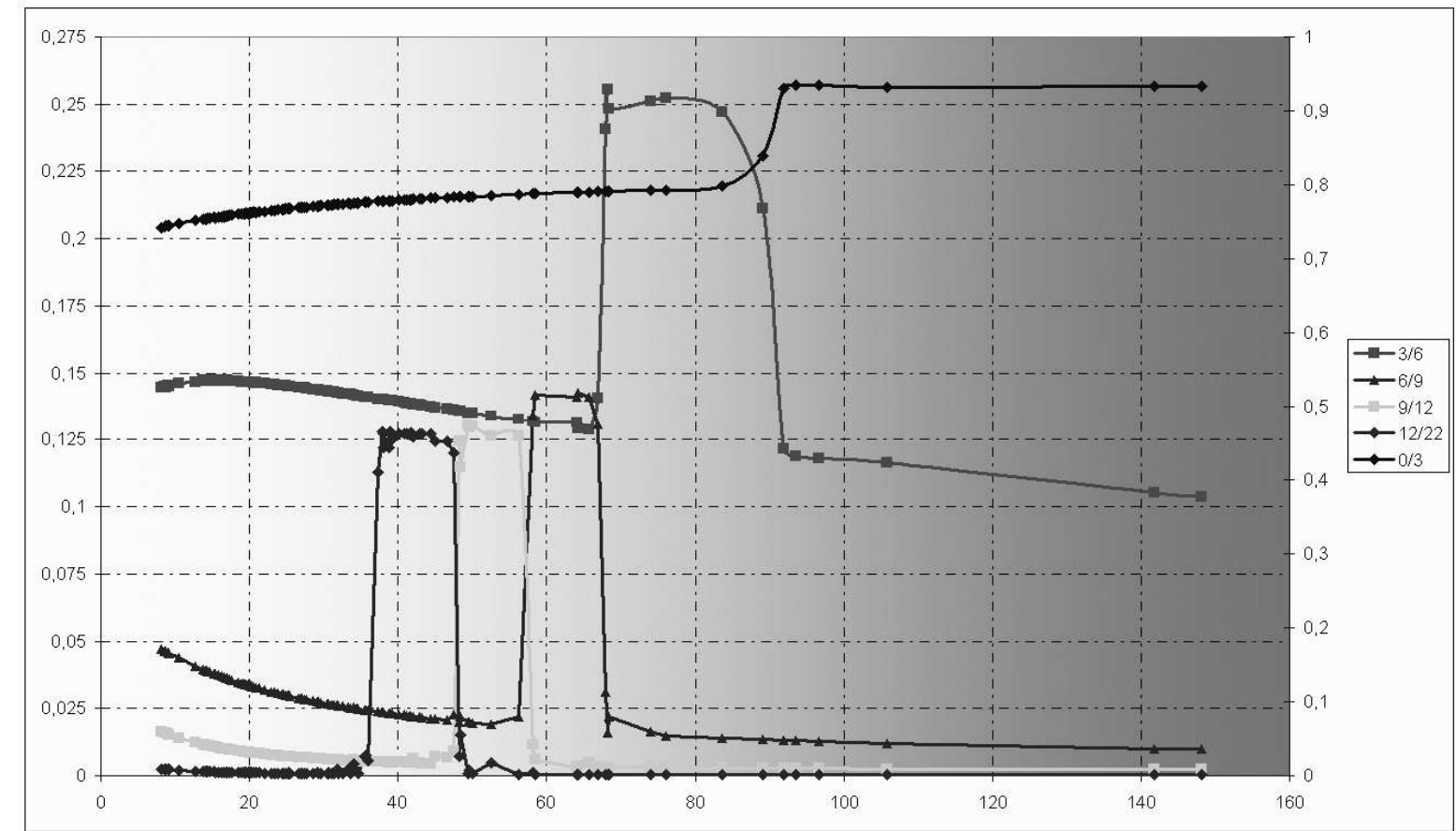

Figure 2. Irregular patterns of individual names deltas in regimes of high correlation.

Figure 2 exhibits CDO tranche deltas with respect to the level of credit spreads computed on 31-August-2005. Nominal is equal to 125. 5-year credit spreads on the $x$-axis are expressed in basis points per annum. Credit deltas of the equity tranche are on right axis. Figure 2 shows that individual credit deltas may actually differ significantly from one name to another.

Such irregular patterns of credit spread deltas will occur whenever pricing models involve some kind of threshold. This is for instance the case with the popular random factor loading approach of Andersen and Sidenius (2005). We want to stress that these patterns of credit spread deltas with respect to the level of credit spreads convey a lot of information. They almost directly show the amount of idiosyncratic gamma risks by the looking at the slope of such a curve. The higher the increase of credit spread deltas with respect to the level of spreads, the higher will the corresponding idiosyncratic gamma ${ }^{39}$.

\footnotetext{
${ }^{38}$ There is always a matter of norm. Here the reference is the credit default swap index. One has also to care about the notional of the tranche in certain cases.

${ }^{39}$ Such a reasoning is not rigorous and holds at the first order. However, the approximation is usually quite good.
} 
The increased efficiency of hedging at a name level is an important issue for trading desks but, as mentioned above, cannot be fully assessed unless the details of the hedging strategy, including the ways hedging parameters are calibrated are disclosed.

\section{Conclusion}

The wise man bridges the gap by laying out the path by means of which he can get from where he is to where he wants to go. John Pierpont Morgan

This paper dealt with the usefulness of pricing and hedging models for the risk management of synthetic CDOs. We show that replicating strategies of CDO tranches can be replicated with self-financing strategies within the basic implementations of the multivariate structural model and of the Markovian loss (or local intensity) model. The former approach is primarily designed to hedge credit spread risks. In the latter approach, focus is put on hedging default risks and may involve a high degree of default contagion. In both cases, credit default swaps are traded to hedge CDO tranches and the involved mathematical tools, such as conditional default probabilities or the use of some martingale representation theorem, are the same. On other grounds these models are strikingly different and each of them could be criticized as too simple and missing some desirable empirical features. The mathematics of the multivariate structural model are well-known ${ }^{40}$. The Gaussian copula model, widely used in the banking industry, may be seen as a one step approximation of the multivariate structural model, especially for investment grade names associated with small default probabilities. This might explain some kind of robustness of the model and lays the path for a better understanding of its limitations. Such an issue has already been studied as far as pricing is concerned, but a formal investigation is still required for hedging.

The overall picture regarding risk management of CDOs looks quite gloomy in the aftermath of the liquidity and credit crisis. Misconceptions about CDO of subprimes, such as the understatement of dependence across minitranches are now well-known and discussed (see Crouhy et al. (2008)) $)^{41}$. If one dares looking at facts eyes wide open, it seems clear that trading desks managing synthetic CDOs had dissimilar performances, which is not surprising, regarding the number of technical issues briefly addressed within this paper. This does not invalidate various quantitative developments in the credit correlation field. According to K. Popper's evolutionary view of science, researchers look for falsification of a theory or a model which represents the state of the art. For this reason, the burgeoning of credit risk models and more recently of back-testing approaches testifies to a vivid research field rather than rigor mortis. Let us consider the supplementary (and somehow challenging) view of $\mathrm{T}$. Kuhn, "No theory ever solves all the puzzles with which it is confronted at a given time; nor are the solutions already achieved often perfect. On the contrary, it is just the incompleteness and imperfection of the existing data-theory fit that, at any given time, define many of the puzzles that characterize normal science". Whether it is time to shift to another paradigm or

\footnotetext{
${ }^{40}$ However, some details are, surprisingly enough, not yet described in the academic literature. We can think of using hedging instruments which vanish before the maturity of the CDO tranche, but are not anymore required after extinction.

${ }^{41}$ We can however notice that it does not invalidate the Gaussian copula model per se, provided that this is applied at the lowest level rather than at the minitranche level. When considering well diversified MBS mezzanine tranches, the idiosyncratic risks are wiped out and only systemic risks such as the price level in the residential mortgage market remains. It is not surprising therefore that the correlation between these minitranches would be much higher than assessed by rating agencies and that senior tranches of ABS were overpriced.
} 
even abandoning the current quantitative finance "research programme" ${ }^{42}$ " is thus still unobvious.

\section{References}

[1] Ammann, M. and B. Brommundt, 2009, Hedging Collateralized Debt Obligations, working paper, University of St Gallen.

[2] Amraoui, S., Cousot, L., Hitier, S., and J-P Laurent, 2009, Pricing CDOs with State Dependent Stochastic Recovery Rates, working paper, ISFA Actuarial School, University of Lyon and BNP Paribas.

[3] Arnsdorf, M., and I. Halperin, 2007, BSLP: Markovian Bivariate Spread-Loss Model for Portfolio Credit Derivatives, working paper, JP Morgan.

[4] Andersen, L., Sidenius, J., and S. Basu, 2003, All your Hedges in One Basket, RISK, November, 67-72.

[5] Andersen, L. and J. Sidenius, 2005, Extensions to the Gaussian Copula: Random Recovery and Random Factor Loadings, Journal of Credit Risk 1(1), 29-70.

[6] Baheti, P. and S. Morgan, 2007, Base Correlation Mapping, Quantitative Research Quarterly, Q1, Lehman Brothers.

[7] Bielecki, T.R., Cousin, A., Crépey, S. and A. Herbertsson, 2010, Pricing and Hedging Portfolio Credit Derivatives in a Bottom-up Model with Simultaneous Defaults, working paper, Evry University.

[8] Bielecki, T.R., Crépey, S., Jeanblanc, M., and M. Rutkowski, 2007, Valuation of Basket Credit Derivatives in the Credit Migrations Environment, Handbook of Financial Engineering, J. Birge and V. Linetsky (eds)., Elsevier.

[9] Bielecki, T.R., Crépey, S., Jeanblanc, M. 2010, Up and Down Credit Risk, Quantitative Finance, Forthcoming.

[10] Bielecki, T.R., Jeanblanc, M., and M. Rutkowski, 2007, Hedging of Basket Credit Derivatives in Default Swap Market, Journal of Credit Risk 3(1), 91-132.

[11] Bielecki, T.R., Vidozzi, A. and L. Vidozzi, 2008, A Markovian Copulae Approach to Pricing and Hedging of Credit Index Derivatives and Ratings Triggered Step-Up Bonds, Journal of Credit Risk 4(1).

[12] Black, F. and J. C. Cox, 1976, Valuing Corporate Securities: Some Effects of Bond Indenture Provisions, Journal of Finance 31(2), 351-367.

[13] Brémaud, P., 1981, Point Processes and Queues: Martingale Dynamics. Springer-Verlag.

[14] Burtschell, X., J. Gregory and J-P. Laurent, 2007, Beyond the Gaussian copula: Stochastic and Local Correlation, Journal of Credit Risk 3(1), 31-62

[15] Burtschell, X., J. Gregory and J-P. Laurent, 2009, A Comparative Analysis of CDO Pricing Models under the Factor Copula Framework, Journal of Derivatives 16(4), 9-37.

[16] Çetin, U., Jarrow, R., Protter, P. and Y. Yildirim, 2004, Modeling Credit Risk with Partial Information. Ann. Appl. Probab 14, 1167-1178

[17] Chen, Z. and P. Glasserman, 2008, Sensitivity Estimates for Portfolio Credit Derivatives using Monte Carlo, Finance and Stochastics 12(4), 507-540.

[18] Coculescu, D., Geman, H. and Jeanblanc, M., 2008, Valuation of Default-Sensitive Claims under Imperfect Information, Finance and Stochastics 12(2), 1432-1122.

[19] Coculescu, D., Jeanblanc, M. and Nikeghbali, A., 2007, Default times, non arbitrage conditions and change of probability measures, working paper, Evry University.

[20] Cont, R., Cousin, A. Crepey, S. and Y. Kan, 2009, Delta Hedging Correlation Risk?, working paper, ISFA Actuarial School, University of Lyon.

[21] Cont, R., Deguest, R. and Y. Kan, 2009, Default Intensities Implied by CDO Spreads: Inversion Formula and Model Calibration, working paper.

\footnotetext{
${ }^{42}$ See Lakatos (1978) regarding the concept of a "research programme" and an attempt to reconcile the views of Popper and of Kuhn. Feyerabend (1975) provides a sharper criticism of Popper's falsification concept.
} 
[22] Cont, R. and Y. Kan, 2008, Dynamic Hedging of Portfolio Credit Derivatives in Top-Down Models, working paper, Columbia University.

[23] Cont, R. and A. Minca, 2008, Recovering Portfolio Default Intensities Implied by CDO Quotes, working paper, Columbia University.

[24] Cousin, A. and M. Jeanblanc, 2010, Hedging Portfolio Loss Derivatives with CDSs, working paper, Evry University.

[25] Cousin, A., Jeanblanc M. and J-P. Laurent, 2009, Hedging CDO tranches in a Markovian environment, book chapter.

[26] Cousin, A., and J-P. Laurent, 2008a, Comparison Results for Exchangeable Credit Risk Portfolios, Insurance: Mathematics and Economics 42(3), 1118-1127.

[27] Cousin, A., and J-P. Laurent, 2008b, Hedging Issues for CDOs, in The Definitive Guide to CDOs, chapter 17, 461-480, G. Meissner (ed.), Risk Books.

[28] Cousin, A. and J-P Laurent, 2008c, An Overview of Factor Modeling for CDO Pricing, in "Frontiers in Quantitative Finance: Credit Risk and Volatility Modeling, R. Cont (ed.), Chapter 7, 185-216, Wiley.

[29] Crouhy, M. G., R. J. Jarrow and S. M. Turnbull, 2008, The Subprime Credit Crisis of 2007, The Journal of Derivatives 16(4), 81-110.

[30] Derman, E., 1999, Regimes of Volatility, Quantitative Strategies Research Notes, Goldman Sachs.

[31] De Servigny, A. and Jobst, N., 2007, The Handbook of Structured Finance, McGraw-Hill.

[32] Ding, J.J. and M. Sherris, 2009, Pricing and Hedging Synthetic CDO tranche Spread Risks, working paper, Australian School of Business, University of New South Wales.

[33] Duffie, D. and Lando, D., 2001, Term Structures of Credit Spreads with Incomplete Accounting Information. Econometrica 69, 633-664.

[34] Dupire, B., 1994, Pricing with a smile, RISK, January, 18-20.

[35] Eckner, A., 2007, Computational Techniques for Basic Affine Models of Portfolio Credit Risk, working paper, Stanford University.

[36] Ehlers, P. and Schönbucher, P. J., 2009, Background filtrations and canonical loss processes for top-down models of portfolio credit risk, Finance and Stochastics 13, 79-103.

[37] Feldhütter, P., 2008, An Empirical Investigation of an Intensity-Based Model for Pricing CDO Tranches, working paper, Copenhagen Business School.

[38] Fermanian, J-D. and O. Vigneron, 2009, On Break-Even Correlation: the Way to Price Structured Credit Derivatives by Replication, working paper.

[39] Feyerabend, P., 1975, Against Method: Outline of an Anarchistic Theory of Knowledge, Humanities Press.

[40] Finger, C., 2009, Testing Hedges Under the Standard Tranched Credit Pricing Model, RiskMetrics Journal 9(1), Winter.

[41] Frey, R., and J. Backhaus, 2007, Dynamic Hedging of Synthetic CDO Tranches with Spread and Contagion Risk, working paper, University of Leipzig.

[42] Frey, R., and J. Backhaus, 2008, Pricing and Hedging of Portfolio Credit Derivatives with Interacting Default Intensities, International Journal of Theoretical and Applied Finance 11(6), 611-634.

[43] Giesecke, K., 2008, Portfolio Credit Risk: Top-Down vs. Bottom-Up Approaches, working paper, Stanford University.

[44] Giesecke, K., and Goldberg, L.R., 2005, A top down approach to multi-name credit, working paper, Stanford University.

[45] Goldberg, L. R. and K. Giesecke, 2004, Forecasting Default in the Face of Uncertainty, Journal of Derivatives 12(1), 14-25.

[46] Gouriéroux, C., and J-P. Laurent, 1996, Estimation of Dynamic Hedge, working paper, CREST.

[47] Gregory, J., and J-P. Laurent, 2008, Practical Pricing of Synthetic CDOs, The Complete Guide to CDOs - Market, Application, Valuation, and Hedging, RISK books.

[48] Guo, X., Jarrow, R.A. and Y. Zeng, 2005, Credit Risk Models with Incomplete Information, Mathematics of Operations Research, To appear. 
[49] Hitier, S. and E. Huber, 2009, CDO Pricing: Copula Implied by Risk Neutral Dynamics, working paper, BNP Paribas.

[50] Houdain, J., 2006, Valorisation et Gestion de Dérivés de Crédit : les CDOs Synthétiques ou la Croissance Exponentielle des Produits de Corrélation, $\mathrm{PhD}$ Thesis, ENS Cachan.

[51] Hull, J., Predescu M., and A. White, 2005, The Valuation of Correlation - Dependent Derivatives Using a Structural Model, working paper, University of Toronto.

[52] Iscoe, I. and A. Kreinin, 2007, Valuation of Synthetic CDOs, Journal of Banking and Finance 31(11), 3357-3376.

[53] Jarrow, R. and P. Protter, 2004, Structural versus Reduced Form Models: A New Information Based Perspective, Journal of Investment Management 2(2), 1-10.

[54] Jobst, N., 2007, An Introduction to the Risk Management of Collateral Debt Obligations, in The Handbook of Structured Finance, A. de Servigny and N. Jobst (eds), McGraw Hill, 295338.

[55] Jeanblanc, M. and Le Cam, Y., 2007, Reduced Form Modelling of Credit Risk, working paper, Evry University.

[56] Jeanblanc, M. and Le Cam, Y., 2009, Immersion Property and Credit Risk Modelling, In Optimality and Risk - Modern Trends in Mathematical Finance, Springer, 99-132.

[57] Joshi, M., and D. Kainth, 2004, Rapid and Accurate Development of Prices and Greeks for nth to Default Swaps in the Li Model, Quantitative Finance, Vol. 4(3), 266-275.

[58] Kakodkar, A., Galiani, S., Jónsson, J. and Gallo, A, 2006, Credit Derivatives Handbook Vol. 2, Merrill Lynch.

[59] Krekel, M., 2008, Pricing distressed CDOs with Base Correlation and Stochastic Recovery, working paper, UniCredit Markets \& Investment Banking.

[60] Kuhn, T. S., The Structure of Scientific Revolutions, 1996, The University of Chicago Press, third edition.

[61] Lakatos, I., 1978, The Methodology of Scientific Research Programmes: Philosophical Papers Volume 1, Cambridge University Press.

[62] Laurent, J-P., 2006, A Note on the Risk Management of CDOs, working paper, ISFA Actuarial School, University of Lyon and BNP Paribas.

[63] Laurent, J-P., Cousin, A., and J-D. Fermanian, 2007, Hedging Default Risk of CDOs in Markovian Contagion Models, working paper, ISFA Actuarial School, University of Lyon, to appear in Quantitative Finance.

[64] Meissner, G., 2008, The Definitive Guide to CDOs. Riskbooks

[65] Meissner, G., Hector, R., and T. Rasmussen, 2008, Hedging CDOs within the Gaussian Copula Framework, in The Definitive Guide to CDOs - Market, Application, Valuation, and Hedging, RISK books.

[66] Morgan, S. and Mortensen, A., 2007, CDO Hedging Anomalies in the Base Correlation Approach, Lehman Brothers, Quantitative Credit Research Quarterly, October, 49-61.

[67] Petrelli, A., Siu, O., Zhang, J., and V. Kapoor, 2006, Optimal Static Hedging of Defaults in $C D O s$, working paper available on www.defaultrisk.com.

[68] Petrelli, A., Zhang, J., Jobst, N., and V. Kapoor, 2007, A Practical Guide to CDO Trading Risk Management, in The Handbook of Structured Finance, A. de Servigny and N. Jobst (eds), McGraw Hill, 339-371.

[69] Popper, K. R., 1992, The Logic of Scientific Discovery, Routledge, Revised Edition.

[70] Rott, M. G., and C. P. Fries, 2005, Fast and Robust Monte Carlo CDO Sensitivities, working paper.

[71] Schloegl, L., Mortensen A. and Morgan, S., 2008, Strange Risk in a Correlation Model, Lehman Brothers, Quantitative Credit Research Quarterly, Q1, 1-7.

[72] Turc, J., Benhamou, D., Herzog B., and M. Teyssier, 2006, Pricing Bespoke CDOs: Latest Developments, Quantitative Strategy, Société Générale.

[73] Walker, M.B., 2008, The Static Hedging of CDO Tranche Correlation Risk, working paper, University of Toronto. 